\title{
Acid ceramidase of macrophages traps herpes simplex virus in multivesicular bodies and protects from severe disease
}

Judith Lang (1) ${ }^{1}$, Patrick Bohn ${ }^{1}$, Hilal Bhat ${ }^{1}$, Holger Jastrow ${ }^{2,3}$, Bernd Walkenfort ${ }^{3}$, Feyza Cansiz ${ }^{1}$, Julian Fink ${ }^{4}$, Michael Bauer (1) ${ }^{5}$, Dominik Olszewski ${ }^{5}$, Ana Ramos-Nascimento ${ }^{6}$, Vikas Duhan ${ }^{1}$, Sarah-Kim Friedrich ${ }^{1}$, Katrin Anne Becker ${ }^{7}$, Adalbert Krawczyk ${ }^{8,9}$, Michael J. Edwards ${ }^{10}$, Andreas Burchert ${ }^{11}$, Magdalena Huber ${ }^{12}$, Justa Friebus-Kardash ${ }^{1}$, Joachim R. Göthert ${ }^{13}$, Cornelia Hardt ${ }^{1}$, Hans Christian Probst (D) ${ }^{14}$, Fabian Schumacher (1) ${ }^{7,15}$, Karl Köhrer (1) ${ }^{16}$, Burkhard Kleuser ${ }^{15}$, Eduard B. Babiychuk ${ }^{17}$, Beate Sodeik (D) 6,18, Jürgen Seibel ${ }^{4}$, Urs F. Greber (10 5 ${ }^{5}$, Philipp A. Lang ${ }^{19}$, Erich Gulbins (1D ${ }^{7,10,20}$ \& Karl S. Lang,20凶

Macrophages have important protective functions during infection with herpes simplex virus type 1 (HSV-1). However, molecular mechanisms that restrict viral propagation and protect from severe disease are unclear. Here we show that macrophages take up HSV-1 via endocytosis and transport the virions into multivesicular bodies (MVBs). In MVBs, acid ceramidase (aCDase) converts ceramide into sphingosine and increases the formation of sphingosine-rich intraluminal vesicles (ILVs). Once HSV-1 particles reach MVBs, sphingosine-rich ILVs bind to HSV-1 particles, which restricts fusion with the limiting endosomal membrane and prevents cellular infection. Lack of aCDase in macrophage cultures or in Asah $1^{-1-}$ mice results in replication of HSV-1 and Asah ${ }^{-1-}$ mice die soon after systemic or intravaginal inoculation. The treatment of macrophages with sphingosine enhancing compounds blocks HSV-1 propagation, suggesting a therapeutic potential of this pathway. In conclusion, aCDase loads ILVs with sphingosine, which prevents HSV-1 capsids from penetrating into the cytosol.

\footnotetext{
${ }^{1}$ Institute of Immunology, University of Duisburg-Essen, Hufelandstr. 55, Essen D-45147, Germany. ${ }^{2}$ Institute of Anatomy, University of Duisburg-Essen, Hufelandstr. 55, Essen D-45147, Germany. ${ }^{3}$ Institut for Experimental Immunology and Imaging, Imaging Center Essen, Electron Microscopy Unit, University of Duisburg-Essen, Hufelandstr. 55, Essen D-45147, Germany. ${ }^{4}$ Institute of Organic Chemistry, Julius-Maximilians University of Würzburg, Am Hubland, Würzburg D-97074, Germany. ${ }^{5}$ Department of Molecular Life Sciences, University of Zurich, Winterthurerstr. 190, CH-8057 Zurich, Switzerland. ${ }^{6}$ Institute of Virology, Hannover Medical School, Carl-Neuberg-Str. 1, Hannover D-30625, Germany. ${ }^{7}$ Institute of Molecular Biology, University of Duisburg-Essen, Hufelandstr. 55, Essen D-45147, Germany. ${ }^{8}$ Institute for Virology, University of Duisburg-Essen, Hufelandstr. 55, Essen D-45147, Germany. ${ }^{9}$ Department of Infectious Diseases, University Hospital of Essen, University of Duisburg-Essen, Hufelandstr. 55, Essen D-45147, Germany. ${ }^{10}$ Department of Surgery, University of Cincinnati, Cincinnati, OH, USA. ${ }^{11}$ Department of Hematology, Oncology and Immunology, University Hospital Giessen and Marburg, Campus Marburg, Baldingerstr., Marburg D-35043, Germany. ${ }^{12}$ Institute of Medical Microbiology and Hospital Hygiene, Philipps-University Marburg, Hans-Meerwein Str. 2, Marburg D-35043, Germany. ${ }^{13}$ Department of Hematology, West German Cancer Center, University Hospital of Essen, University of Duisburg-Essen, Hufelandstr. 55, Essen D-45147, Germany. ${ }^{14}$ Institute of Immunology, University Medical Center Mainz, Langenbeckstr. 1, Mainz D-55131, Germany. ${ }^{15}$ Institute of Nutritional Science, University of Potsdam, Arthur-Scheunert Allee 114-116, Nuthetal D-14558, Germany. ${ }^{16}$ Biological and Medical Research Center (BMFZ), Heinrich-Heine-University, Universitätsstr. 1, Düsseldorf D-40225, Germany. ${ }^{17}$ Institute of Anatomy, University of Bern, Baltzerstr. 4, CH-3012 Bern, Switzerland. ${ }^{18}$ Cluster of Excellence RESIST (EXC 2155), Hannover Medical School, Carl-Neuberg-Str. 1, Hannover D-30625, Germany. ${ }^{19}$ Department of Molecular Medicine II, Medical Faculty, Heinrich Heine University, Universitätsstr. 1, Düsseldorf D-40225, Germany. ${ }^{20}$ These authors jointly supervised: Erich Gulbins, Karl S. Lang. ${ }^{凶}$ email: karlsebastian.lang@uk-essen.de
} 
M acrophages are one of the most important components of the innate immune system. With their strong phagocytic capacity they engulf pathogens early after their invasion and inactivate them by proteolysis ${ }^{1}$. Especially during virus infection, macrophages are the key effector cells of innate immunity. Mice lacking macrophages are highly susceptible for instance to mouse hepatitis virus, lymphocytic choriomeningitis virus and vesicular stomatitis virus and show overwhelming viral propagation ${ }^{2-4}$. The contribution of macrophages to control of systemic infection was linked to their capacity to internalize infectious particles and limit their propagation in a type I interferon (IFN-I) dependent manner, which leads to upregulation of the antiviral genes $\mathrm{Mx}, \mathrm{OAS}, \mathrm{RNase} \mathrm{L}$ and Protein kinase $\mathrm{R}^{4}$. While these antiviral mechanisms require hours after infection to become active, macrophages also exhibit robust antiviral suppression already minutes after virus inoculation ${ }^{4-6}$, suggesting the presence of preexisting IFN-I-independent antiviral mechanisms. However, these mechanisms are poorly defined.

Herpes simplex virus type 1 (HSV-1) is an enveloped DNA virus, which can replicate in several different cell types ${ }^{7}$. After binding to a target cell, HSV-1 either fuses directly with the plasma membrane or is taken up into endosomes of the cell ${ }^{8,9}$. Within these endosomes fusion of the outer limiting membrane with HSV-1 membrane leads to infection of the cells in a $\mathrm{pH}$ dependent manner ${ }^{10}$. Upon penetration, the HSV-1 capsid traffics on microtubules to the nucleus ${ }^{11,12}$, uncoats its DNA genome at the nuclear pore complex ${ }^{13,14}$, and delivers the genome to the nucleus for replication and assembly of capsids ${ }^{15,16}$. Newly formed capsids bud through the nuclear envelope $\mathrm{e}^{17}$ and exit the infected cell through the secretory pathway or by cell lysis ${ }^{18,19}$. In an HSV-1 infection model, macrophages play an essential role ${ }^{20}$. This is linked to the high IFN-I sensitivity of HSV-1, which limits the replication of HSV-1 DNA and the production of newly formed capsids ${ }^{21,22}$. Interestingly even in the absence of IFN-I replication of HSV-1 is restricted in macrophages, a phenomenon that could be explained by limited uptake of HSV-1 into macrophages or an aggravated or limited infection of the macrophage after HSV-1 has entered the endosome. Whether indeed macrophage-specific mechanisms regulate HSV-1 infection remains unknown.

Mulitvesicular bodies (MVBs) are complex membrane rich organelles of the cytoplasm, which contain high numbers of vesicles. These so called intraluminal vesicles (ILVs) are formed by invagination and budding from the outer limiting membranes (OLMs) into the MVB lumen and play a crucial role in sorting, degrading, and recycling of proteins ${ }^{23}$. Sphingolipids are enriched in MVBs and are involved in the MVB biogenesis and the regulation of their functions ${ }^{24}$. Thereby sphingolipids modulate diverse cellular processes, including proliferation, differentiation, apoptosis, signal transduction, and membrane trafficking ${ }^{25-27}$. Modulation of biophysical membrane properties such as fluidity, polarity, vesicle formation, and deformation is one major factor of the regulatory capacity of sphingolipids ${ }^{28}$. A key enzyme in sphingolipid metabolism in MVBs is the acid ceramidase (aCDase) ${ }^{29-31}$. It catalyzes the degradation of ceramide into sphingosine and free fatty acid, which modulates the shape and the charge of membranes. Whether this process can modulate HSV-1 entry remains unknown.

Here we analyze whether the aCDase dependent modulation of membrane properties can affect HSV-1 entry after uptake into macrophages. In a model of HSV-1 infection in bone marrow derived murine macrophages (BMDMs) we found that strong activity of aCDase blocks the fusion of the HSV-1 membrane with the outer limiting membrane of MVBs. Antiviral activity of aCDase is independent of IFN-I, but aCDase expression is dependent on IRF8 during macrophage development. In vivo, expression of aCDase in macrophages limits early steps of HSV-1 propagation and protects mice from severe outcome of infection.

\section{Results}

Macrophages control HSV-1 infection. To prove the importance of macrophages during herpes simplex virus type 1 (HSV-1) control in vivo, we infected C57BL/6 mice intravenously with HSV- 1 and then analyzed the distribution of virus in the liver by histology. Naïve mice and HSV-1 infected macrophage-depleted mice served as controls. $1 \mathrm{~h}$ after infection most of the virus inoculum co-localized with $\mathrm{F} 4 / 80^{+}$macrophages within the liver (Fig. 1a), suggesting that macrophages are equipped to take up HSV-1 quickly after systemic administration. Naïve mice did not show HSV-1 staining (Fig. 1a) and macrophage-depleted mice showed no $\mathrm{F} 4 / 80^{+}$cells in spleen and liver and no co-localization (Supplementary Fig. 1) ${ }^{4}$. To obtain insights on the functional role of macrophages we treated C57BL/6 mice with clodronateliposomes to deplete macrophages. Control mice were treated with control-liposomes and all mice were infected with HSV-1. In the presence of macrophages, HSV-1 replication was nearly undetectable $24 \mathrm{~h}$ after infection (Fig. 1b, c). In strong contrast, HSV-1 showed overwhelming replication in the absence of macrophages (Fig. 1b, c). Enhanced replication of HSV-1 correlated with production of infectious HSV-1 in liver and spleen of macrophage deficient mice (Fig. 1d). In line with overwhelming virus propagation, macrophage deficient mice developed liver cell damage and died quickly after infection (Fig. 1e, f). To analyze the importance of macrophages during a natural infection route, we infected mice intravaginal with HSV- 1 and analyzed local replication of HSV-1 and outcome of the infection. Local HSV-1 replication and production of infectious particles was comparable between control and macrophage-depleted mice (Fig. 1g, h). However, macrophage-depleted mice died after intravaginal infection while control mice were protected (Fig. 1i). This suggests that macrophages in the vagina do not limit early local HSV-1 replication, while, macrophages are critical for the systemic control of HSV-1. In conclusion, we found that macrophages do play an essential role to protect from systemic HSV-1 propagation.

aCDase in multivesicular bodies traps HSV-1. As elucidated in Fig. 1, macrophages are of high importance to limit HSV-1 propagation during systemic infection. Following this, we aimed to investigate antiviral mechanisms that are specific to macrophages. To get insights into the macrophage-specific antiviral mechanism we generated bone marrow derived macrophages (BMDMs) and compared their antiviral activity to isolated alveolar fibroblasts. HSV-1 propagated strongly in fibroblasts and infectious HSV-1 was released into the supernatant (Fig. 2a, b). In contrast to fibroblasts, HSV-1 showed limited propagation in macrophages and infectious particles were hardly measurable in the supernatants (Fig. 2a, b). Even higher infection doses up to MOI 1 did not result in productive infection in cultured macrophages (Fig. 2b). Treatment with interferon- $\alpha 4$ (IFN- $\alpha 4$ ) reduced the infectious particles (Fig. 2b), however the striking difference in virus production between fibroblasts and macrophages remained. These results suggest that administration of type I interferon (IFN-I) cannot induce the strong antiviral capacities of macrophages in fibroblasts. While there are multiple differences between macrophages and fibroblasts, we hypothesized that macrophages have specific antiviral protection mechanisms independent of the presence of IFN-I.

To explore potential antiviral mechanisms we performed microarray analysis to compare cellular pathways between macrophages and fibroblasts. The strongest difference between 
a
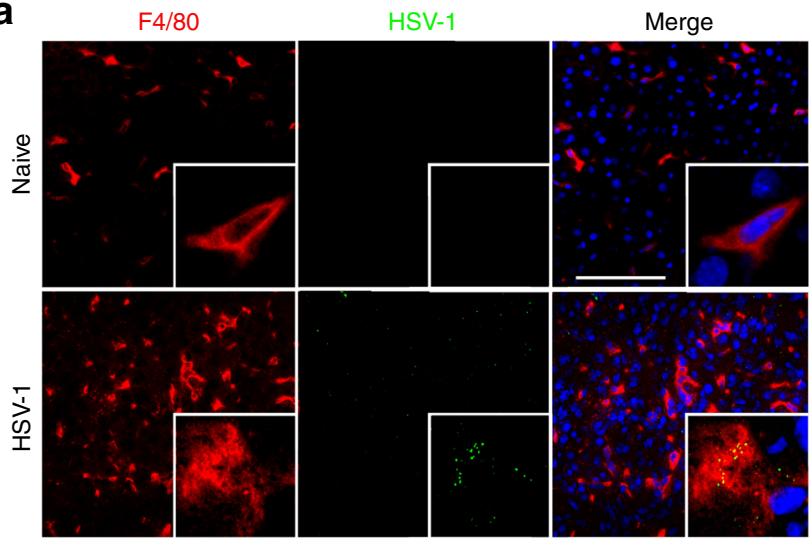

b
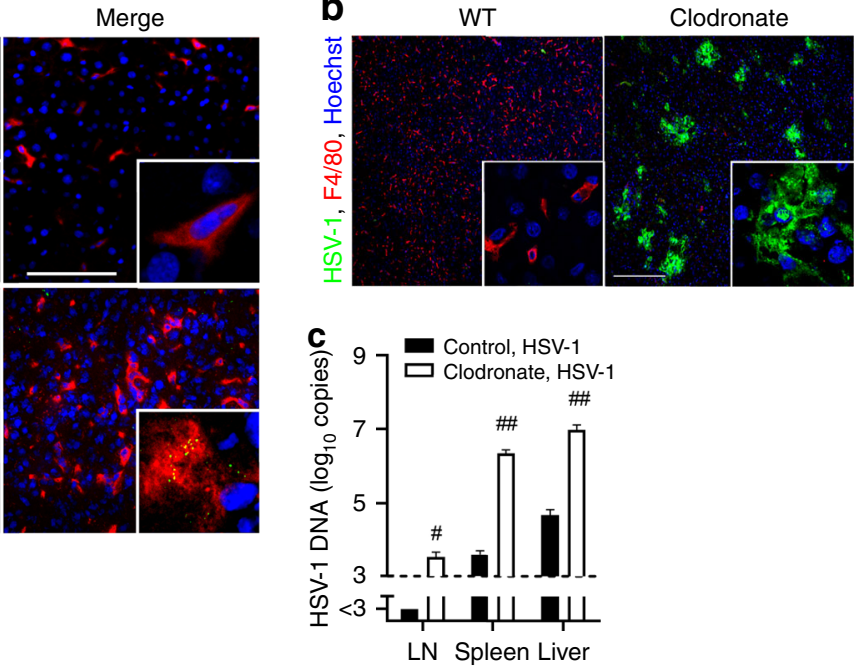
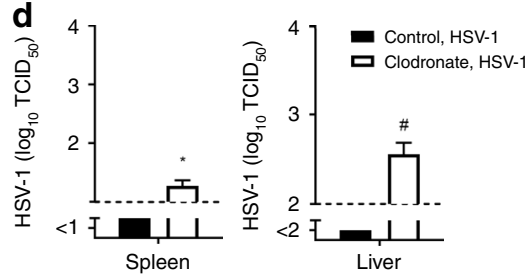

f
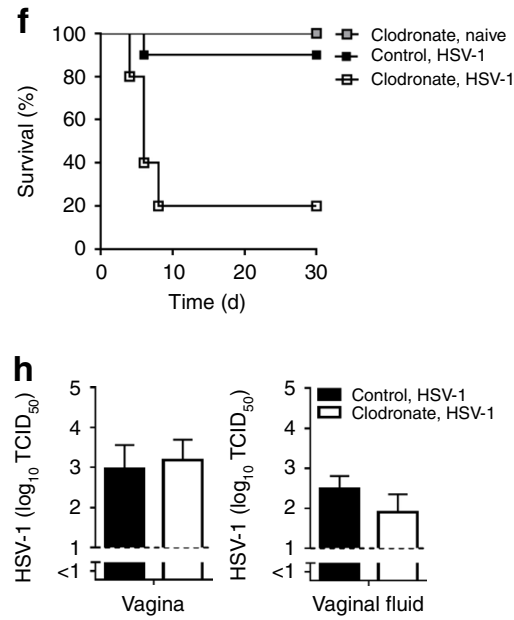

e
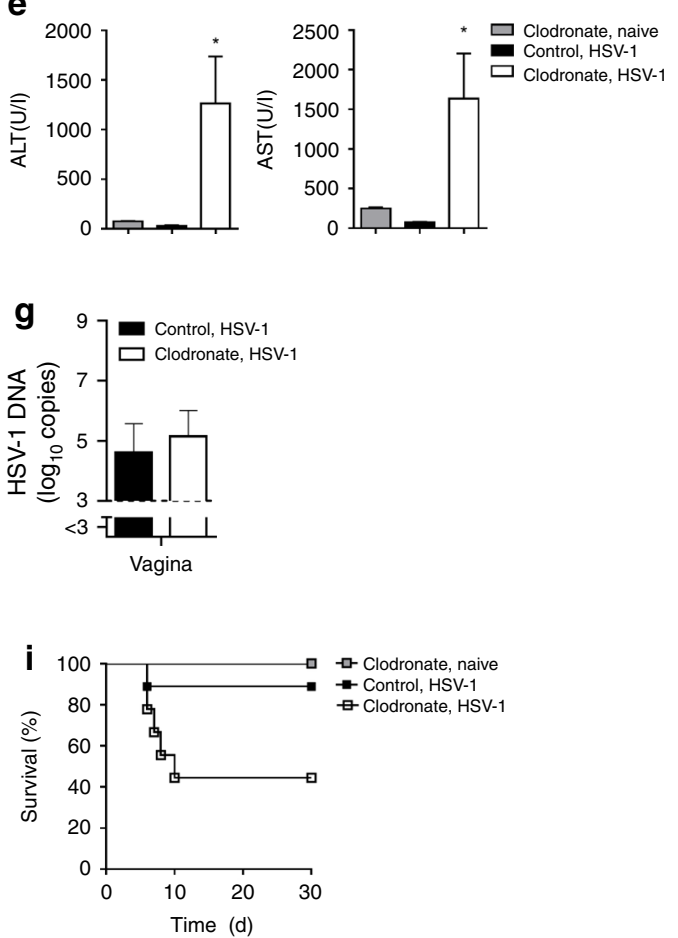

Fig. 1 Macrophages control HSV-1 infection. a Immunofluorescence of livers from wild-type (WT) mice that were not or were infected with $8 \times 10^{7}$ tissue culture infection dose $50\left(\right.$ TCID $\left._{50}\right) \mathrm{HSV}-1$ and analyzed after $1 \mathrm{~h}(n=3$, blue represents Hoechst staining, scale bar $100 \mu \mathrm{m})$. b-d Immunofluorescence of livers $(\mathbf{b}, n=8$, scale bar $200 \mu \mathrm{m})$, real-time polymerase chain reaction (RT-PCR) of lymph nodes (LN), spleens and livers (c, $n=12-14$, two-tailed student's $t$-test) and TCID 50 of spleens and livers ( $\mathbf{d}, n=8$, two-tailed student's $t$-test) from WT mice that were pretreated with control-liposomes and WT mice that were pretreated with clodronate-liposomes (day -3), infected with $6 \times 10^{6} \mathrm{TCID}_{50} \mathrm{HSV}-1$ and analyzed after $24 \mathrm{~h}$. e, $\mathbf{f}$ Alanine transaminase (ALT) and aspartate transaminase (AST) activity (e; $n=10$, one-way ANOVA [Tukey's multiple comparison]) and survival of mice (f) that were treated with clodronate-liposomes or control liposomes $(n=10$; day -3$)$ and then infected with $6 \times 10^{5} \mathrm{TCID}_{50} \mathrm{HSV}-1$ or left uninfected $(n=5 ; p=0.0006$, Logrank [Mantel-Cox] test). $\mathbf{g}$, h RT-PCR of vagina $(\mathbf{g})$ and $\mathrm{TCID}_{50}$ of vagina and vaginal fluid (h) of mice that were treated with clodronate-liposomes or control-liposomes (day -3) and then infected with $2 \times 10^{7} \mathrm{TCID}$ o HSV-1 intravaginally, analyzed after $24 \mathrm{~h}(n=6$, two-tailed student's $t$-test). i Survival of mice that were treated with clodronate-liposomes or control-liposomes (day -3 ) and then infected with $2 \times 10^{7} \mathrm{TCID}_{50} \mathrm{HSV}_{-1}$ intravaginally $(n=9)$, or left uninfected $\left(n=5 ; p=0.0372\right.$, Log-rank [Mantel-Cox] test). All data are shown as mean \pm SEM. ${ }^{\star} p \leq 0.05,{ }^{\star \star} p \leq 0.01,{ }^{\#} p \leq 0.001,{ }^{\# \#} p \leq 0.0001$.

fibroblasts and macrophages was the high expression of endosomal, lysosomal and vesicular transport pathways in macrophages (Fig. 2c). From these data, we considered it likely that in macrophages the vesicular trafficking of HSV-1 might be extraordinary, and therefore restricts a productive infection. To directly visualize HSV-1 entry in macrophages and fibroblasts, we performed electron microscopy of macrophages and fibroblasts, which were infected with HSV-1 (MOI 250) for $30 \mathrm{~min}$. We chose $30 \mathrm{~min}$, as this is the time that HSV-1 requires to enter cells and first incoming capsids to reach the nuclear envelope of fibroblasts or epithelial cells ${ }^{11,32-34}$. Indeed, $30 \mathrm{~min}$ after infection we detected first viral capsids at the nuclear envelope of fibroblasts, but no newly produced viral capsids (Supplementary Fig. 2). Therefore we concluded that $30 \mathrm{~min}$ is an ideal time point to 
a

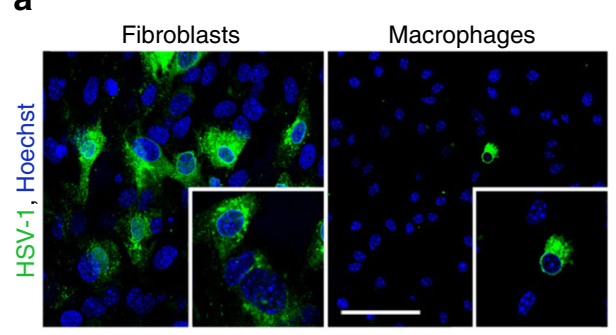

b

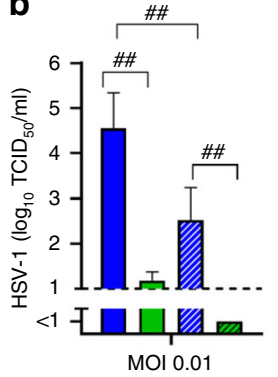

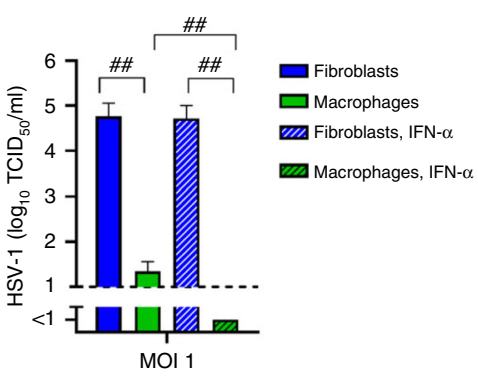

e

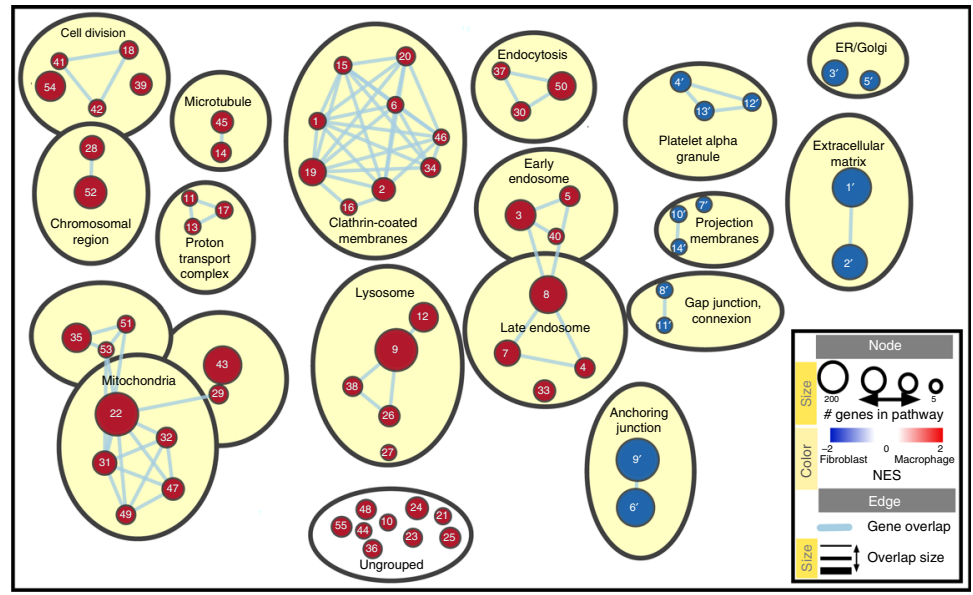

d

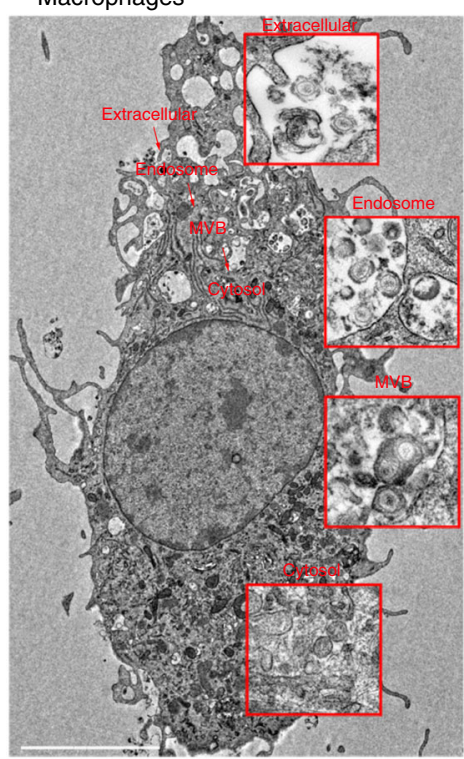

Fibroblasts

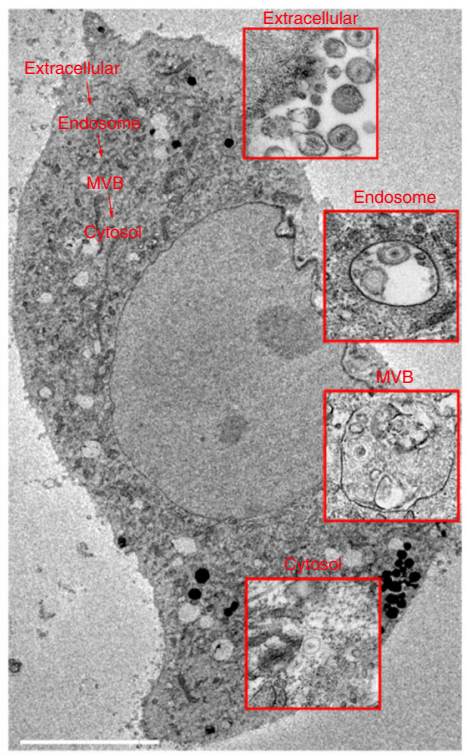

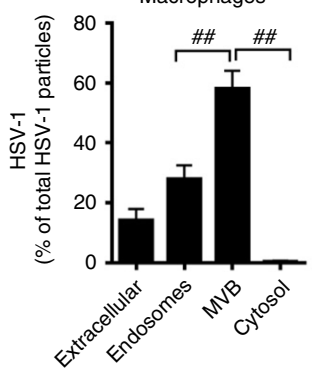

f
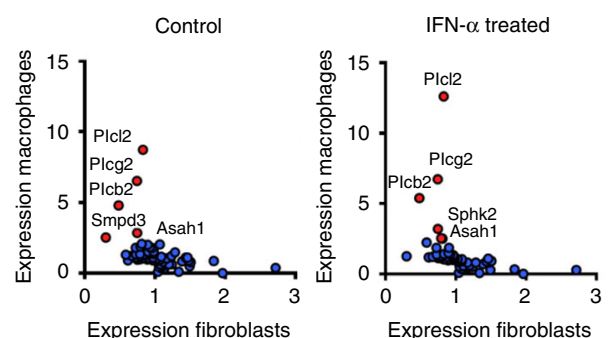

9

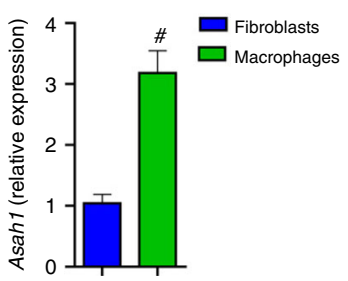

h

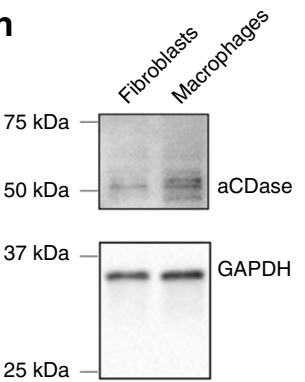

analyze the early internalization and intracellular distribution of HSV-1 within the cell. As expected, we found in macrophage and fibroblast cell cultures HSV-1 virions close to the plasma membrane (Fig. 2d). In macrophages, about $30 \%$ of all particles were found in endosomes and significantly more particles, about $50 \%$ of all particles, were found in multivesicular bodies
(Fig. 2d, e). We could hardly detect viral capsids in the cytosol of macrophages (Fig. 2d, e). In fibroblasts, HSV-1 was detectable in vesicular compartments like early endosomes, late endosomes, MVBs and/or lysosomes (Fig. 2d). In addition electron microscopy revealed cytosolic capsids after $30 \mathrm{~min}$ of infection (Fig. 2d), indicating fusion of the viral envelopes with host membrane and 
Fig. 2 aCDase in multivesicular bodies traps HSV-1. a, b Immunofluorescence (a) and tissue culture infection dose $50\left(T C I D_{50}\right)$ from supernatants (b) of fibroblasts or bone marrow derived macrophages (BMDMs) that were infected with herpes simplex virus type 1 (HSV-1) at a multiplicity of infection (MOI) of 10 and stained after $6 \mathrm{~h}(\mathbf{a}, n=4$; scale bar $50 \mu \mathrm{m})$ or MOI 0.01 or MOI 1 and supernatant was collected after $24 \mathrm{~h}$ (b, $n=8$; one-way ANOVA [Tukey's multiple comparison]). c Gene set enrichment analysis (GSEA) of microarrays showing gene sets enriched in macrophages (red) vs. gene sets enriched in fibroblasts (blue). See Supplementary Table 1a, b for gene sets, ranking and grouping. NES: normalized enrichment score. ER: endoplasmic reticulum. d, e Representative electron microscopic images (d) and quantification (e) of wild-type (WT) BMDMs and/or fibroblasts infected with HSV-1 (MOI 250) analyzed after 30 min (BMDMs: $n=77$ images from three independent experiments, scale bar $5 \mu$ m; Fibroblasts: $n=106$ images from three independent experiments, scale bar $5 \mu \mathrm{m}$, one-way ANOVA [Tukey's multiple comparison]). Overview of one macrophage and one fibroblast is shown. Details show specific compartments containing HSV-1. MVB: multivesicular bodies. f: Expression of membrane-modulating proteins analyzed from microarrays of fibroblasts and macrophages with or without $50 \mathrm{U} / \mathrm{ml}$ interferon- $\alpha 4$ (IFN- $\alpha 4$ ) treatment. $\mathbf{g}$ Real-time polymerase chain reaction (RT-PCR) for Asah1 mRNA expression of fibroblasts and macrophages from WT mice $(n=6$, two-tailed student's $t$-test). $\mathbf{h}$ Western Blot for aCDase protein expression of fibroblasts and macrophages from WT mice (1 of 2 is shown). All data are shown as mean \pm SEM. ${ }^{\star} p \leq 0.05,{ }^{\star \star} p \leq 0.01,{ }^{\#} p \leq 0.001,{ }^{\# \#} p \leq 0.0001$.

successful capsid delivery into the cytosol. From these results we concluded that after HSV-1 is internalized by macrophages, it passes early and late endosomes and accumulates in MVBs but cannot deliver its capsid through the outer limiting membrane to the cytosol. This phenomenon might be responsible for limited productive infection in macrophages.

To determine why HSV-1 fails to release its capsid through the outer limiting MVB membrane in macrophages, we analyzed our microarray data for differences in membrane-modulating proteins. Strikingly we found overexpression of phospholipase C subtypes, neutral sphingomyelinase (Nsm, Smpd3) and acid ceramidase (aCDase, Asah1) in macrophages (Fig. 2f) in comparison to fibroblasts. Differential expression of these proteins was independent of IFN-I (Fig. 2f). Using real-time polymerase chain reaction (RT-PCR) and Western blot analysis we confirmed the high expression of Asah1 mRNA and cleaved, i.e., activated, aCDase protein in macrophages compared to fibroblasts (Fig. $2 \mathrm{~g}, \mathrm{~h}$, Supplementary Fig. 7). From these experiments we concluded that macrophages accumulate HSV-1 in MVBs without being productively infected. This strong antiviral activity of macrophages correlated with aCDase expression.

aCDase limits HSV-1 infection and prevents disease. Since we identified that aCDase is a membrane modulating molecule that is highly expressed in macrophages, we next analyzed the antiviral activity of aCDase. WT macrophages showed limited propagation of HSV-1 (Fig. 3a, b). In strong contrast macrophages that lack aCDase $\left(A s a h 1^{-l-}\right)$ showed strong propagation of HSV-1 (Fig. 3a, b). Next we tested the role of aCDase in vivo. We infected WT and Asah $1^{-1-}$ mice with HSV-1. In Asah $1^{-1-}$ mice, HSV - 1 showed overwhelming propagation after $12 \mathrm{~h}$ to 3 days (Fig. 3c, d) and infectious particles were produced in spleen and liver of $A s a h 1^{-1-}$, but not control mice (Fig. 3e). Next we aimed to determine the importance of Asah1 in relation to the known anti-HSV-1 effectors type I interferon (IFN-I) ${ }^{35}$ and SAMHD ${ }^{36}$. Therefore we infected mice, which show limited production of IFN-I (MyD88 $8^{-1-} x$ Trif $^{-1-} x$ Cardif $^{-1-}$ mice), limited response to IFN-I (Ifnar-l- mice) and mice, which lack SAMHD1 (Samhd1 ${ }^{-1-}$ mice), with HSV-1. Similarly to Asah1 ${ }^{-1-}$ mice, Ifnar $^{-1-}$ mice and $M y D 88^{-1-} x$ Trif $^{-1-} x$ Cardif $^{-1-}$ mice showed highly elevated HSV-1 DNA in lymph node, spleen and liver, when compared to control mice (Supplementary Fig. 3). In Samhd1 $1^{-1-}$ mice HSV-1 DNA levels were elevated in the liver (Supplementary Fig. 3). In Ifnar ${ }^{-1-}$ mice and $M y D 88^{-1-} x$ Trif $^{-1-} \times$ Cardif $^{-1}$ mice, but not in Samhd1 ${ }^{-1-}$ mice, we detected infectious HSV-1 particles in liver and spleen after HSV-1 infection, which was comparable to HSV-1 production in Asah $1^{-1-}$ mice (Fig. 3f). From these data we considered aCDase to be an important antiviral effector mechanism.

To determine the macrophage-specific role of aCDase we generated bone marrow chimeras, in which $A s a h 1^{-/-}$bone marrow was transplanted into macrophage-depleted WT mice. In these mice macrophages repopulate from $A s a h 1^{-1-}$ bone marrow. Chimeric mice with aCDase-deficient macrophages showed enhanced HSV-1 copies when compared to control mice (Fig. 3g), suggesting that indeed aCDase in macrophages contributed to control of HSV-1. To determine the overall importance of aCDase during systemic and local infection we infected Tamoxifen treated Cre-ER Asahl $1^{f l / f l}$ (inducible aCDase-deficient mice) with HSV-1. aCDase deficient Cre-ER Asah $1^{f l / f l}$ mice died quickly after systemic and local intravaginal infection (Fig. 3h, i). Therefore we conclude that aCDase is an important effector mechanism against HSV-1, which acts in macrophages.

Sphingosine protects against HSV-1 infection. After analyzing aCDase deficiency, we investigated effects of increasing sphingolipid content during HSV-1 infection. From literature it is known that aCDase is usually active in lysosomes and $\mathrm{MVBs}^{29-31,37}$. To test whether aCDase directly interacts with HSV-1, we infected macrophages with HSV-1 and stained for aCDase and HSV-1 after $30 \mathrm{~min}$. HSV -1 and aCDase were in close approximation, however we did not see a direct co-localization of aCDase with HSV-1 (Fig. 4a). This suggests that aCDase is not directly modulating HSV-1, but might modulate cellular components such as MVBs, which affect HSV-1 infection. Functionally, aCDase converts ceramide into sphingosine (Fig. 4b) and we considered that enhancement of sphingosine in MVBs was the reason for viral protection in WT macrophages against HSV-1. Indeed, addition of CDase $30 \mathrm{~min}$ before infection limits HSV-1 propagation, which suggests that the enzymatic function is most relevant for the antiviral activity (Fig. 4c, d).To reveal the role of sphingosine in HSV-1 infection, we treated macrophages with sphingosine (Sph), sphingomyelinase (SMase), and sphingosine kinase inhibitor (SKI-II), which are all involved in sphingolipid pathway and could enhance sphingosine levels ${ }^{38}$. Sphingosine treatment, which strongly elevated total sphingosine levels of primary macrophages and the Raw264.7 macrophage cell line (Supplementary Fig. 4), suppressed propagation of HSV-1 (Fig. 4c-e). Treatment with ceramidase, sphingomyelinase, and sphingosine kinase inhibitor resulted in smaller increases of cellular sphingosine levels and a corresponding suppression of HSV-1 propagation (Fig. 4c-e, Supplementary Fig. 4). From these data we concluded that enhanced amount of sphingosine was most likely the reason for antiviral activity in the presence of aCDase. To determine whether sphingosine acted cell type specific, we tested the antiviral activity of sphingosine in different cell types including human monocytes (THP-1), human cervix carcinoma cells (HeLa) and monkey kidney cells (Vero). To note, HSV-1 infects Vero cells via fusion at the plasma membrane while HeLa cells are infected after HSV-1 is endocytosed ${ }^{10,11,39,40}$. We found that sphingosine and/or SKI-II acted antiviral in undifferentiated THP-1 and HeLa cells (Fig. 4f, g). However we 


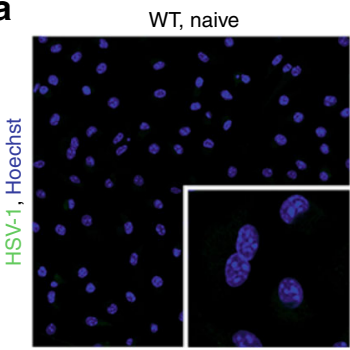

$\mathbf{C}$

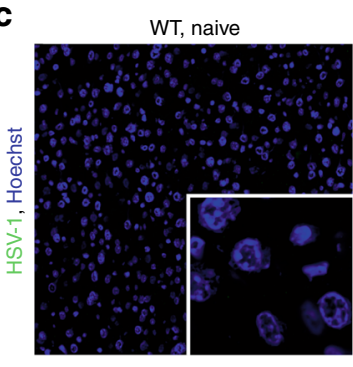

e

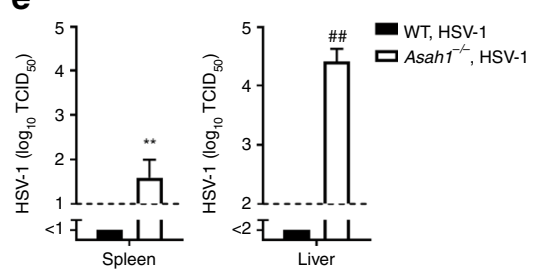

h

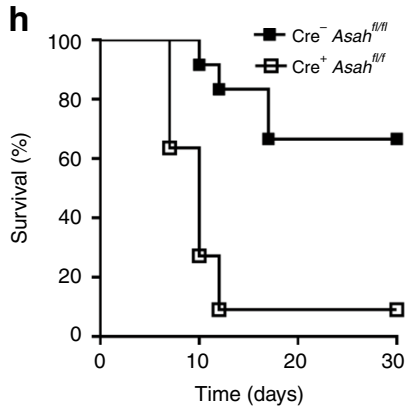

WT

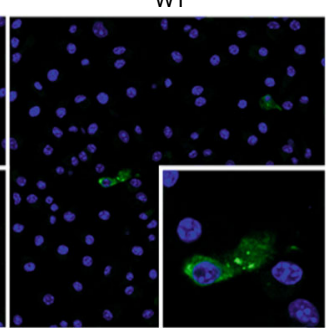

WT

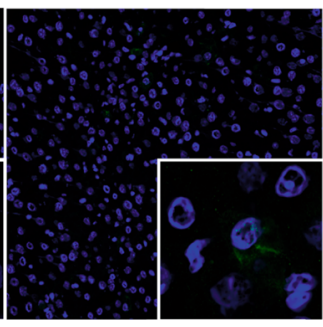

Asah $^{-1-}$

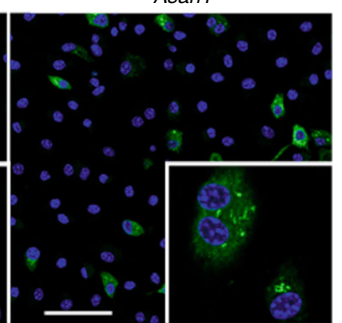

Asah1 $1^{-/}$

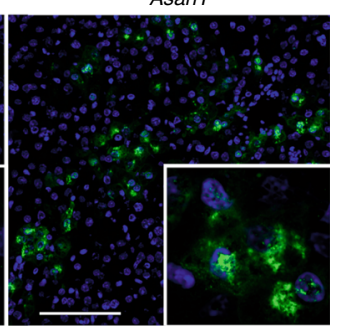

b

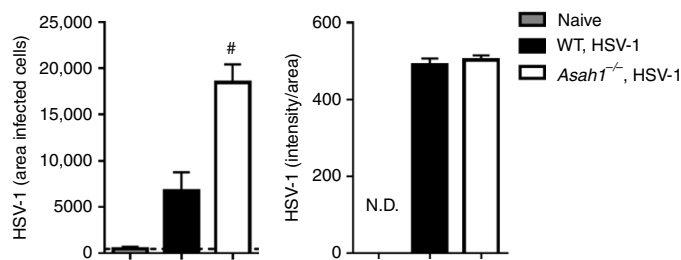

d

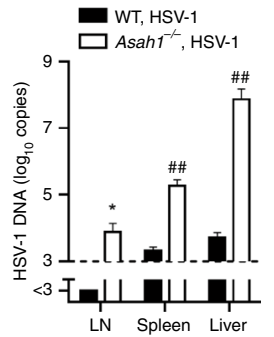

f

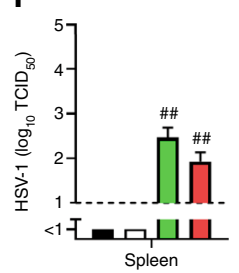

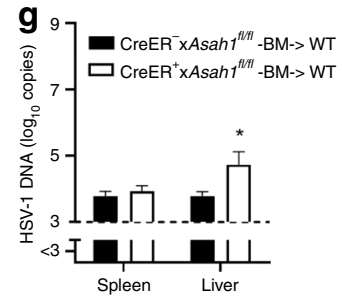

i
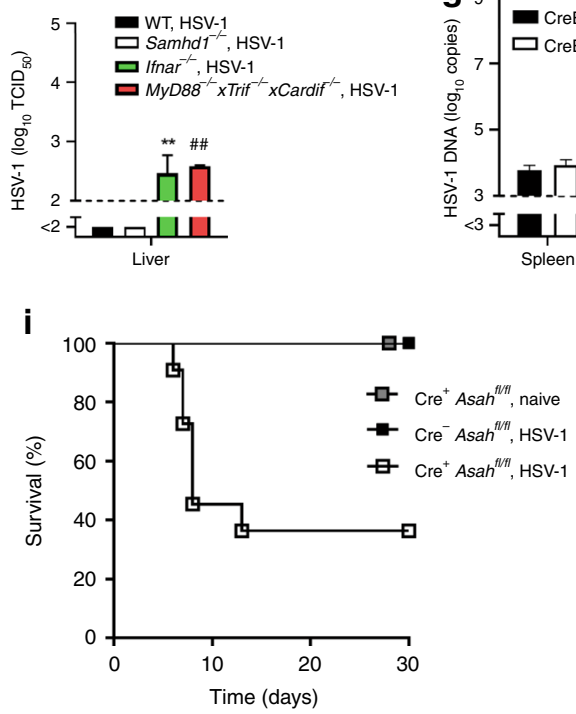

Fig. 3 aCDase limits HSV-1 infection and prevents disease. a, b Representative images (a) and quantification (b) of immunofluorescence from wild-type (WT) and Asah1-/- bone marrow derived macrophages (BMDMs) infected with HSV-1 for $6 \mathrm{~h}$ (multiplicity of infection [MOI] 10, $n=5$, scale bar 50 mm, two-tailed student's $t$-test). N.D.: not done. c Immunofluorescence of livers from WT and Asah1-/- mice that were infected with $7 \times 10^{7}$ plaque forming units (PFU) HSV-1 and analyzed after $12 \mathrm{~h}(n=6-9$, scale bar $100 \mu \mathrm{m})$. d, e Real-time polymerase chain reaction (RT-PCR) of lymph nodes (LN), spleens and livers ( $\mathbf{d}, n=7-10,2$-way ANOVA [Sidak's multiple comparison]) and tissue culture infection dose 50 (TCID 50 , e, $n=4-6$, two-tailed student's $t$-test) of spleens and livers from WT mice and Asah1-/- mice that were infected with $2 \times 10^{6}$ TCID $_{50}$ HSV-1 and analyzed on day 3 . $\mathbf{f}$ TCID 50 of spleens and livers

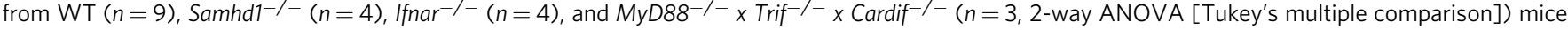
that were infected with $2 \times 10^{6} \mathrm{TCID}_{50} \mathrm{HSV}-1$ and analyzed on day 3. $\mathrm{g}$ RT-PCR of spleens and livers from bone marrow chimera mice receiving Cre Asah $7^{\mathrm{fl} / \mathrm{fl}}$ or $\mathrm{Cre}^{+}$Asahfl/fl bone marrow that were tamoxifen-treated, infected with $6 \times 10^{6} \mathrm{TCID}_{50} \mathrm{HSV}-1$ and analyzed on day 3 ( $n=4-5$, two-tailed

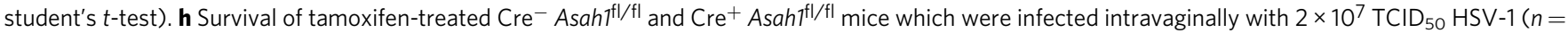
$11-12, p=0.0004$, Log-rank [Mantel-Cox] test). i Survival of tamoxifen-treated $\mathrm{Cre}^{-}$Asahfl/fl and $\mathrm{Cre}^{+}$Asahf $\mathrm{fl}^{\mathrm{f} / \mathrm{fl}}$ mice which were infected intravenously with $5 \times 10^{5}$ TCID $_{50}$ HSV-1 $\left(n=11-13, p=0.0004\right.$, Log-rank [Mantel-Cox] test) or left uninfected $\left(C r e+A s a h 7^{f l} / f l\right.$, naïve; $\left.^{+}=5\right)$. All data are shown as

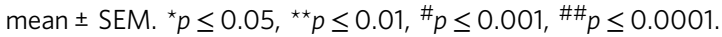

found limited antiviral activity of sphingosine in Vero cells (Fig. 4h), suggesting that the cell type and/or the route of infection might limit the antiviral activity of sphingosine. Taken together we concluded, that sphingosine, which is produced by aCDase in MVBs is antivirally active in macrophages.

Sphingosine-rich intraluminal vesicles trap HSV-1. Next we aimed to determine the mechanism, how aCDase and sphingosine limit HSV-1 infection in macrophages. To this end, we incubated cells for $30 \mathrm{~min}$ with an HSV-1 carrying a fluorescently labeled tegument (HSV-1-VP16-GFP), and stained with an antibody for viral capsids $\left(\mathrm{VP} 5^{41}\right)$, which recognizes the uncoated capsid after virus membrane fusion. In this experimental setup capsid is only visible after dissociation of the tegument ${ }^{11}$. We mainly observed HSV-1-VP16-GFP puncta, in WT macrophages (Fig. 5a, b), indicating that the HSV-1 capsids 
a
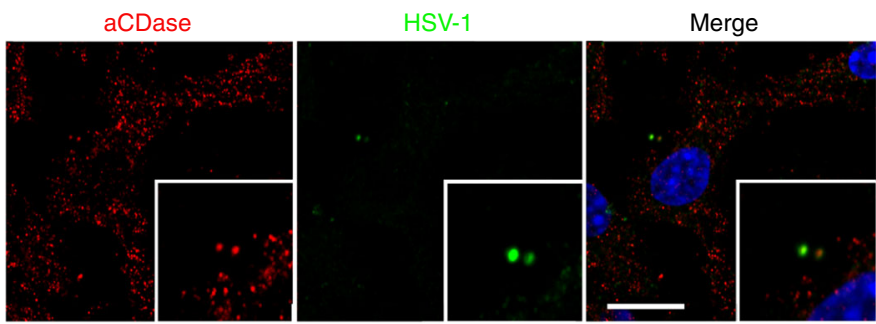

C
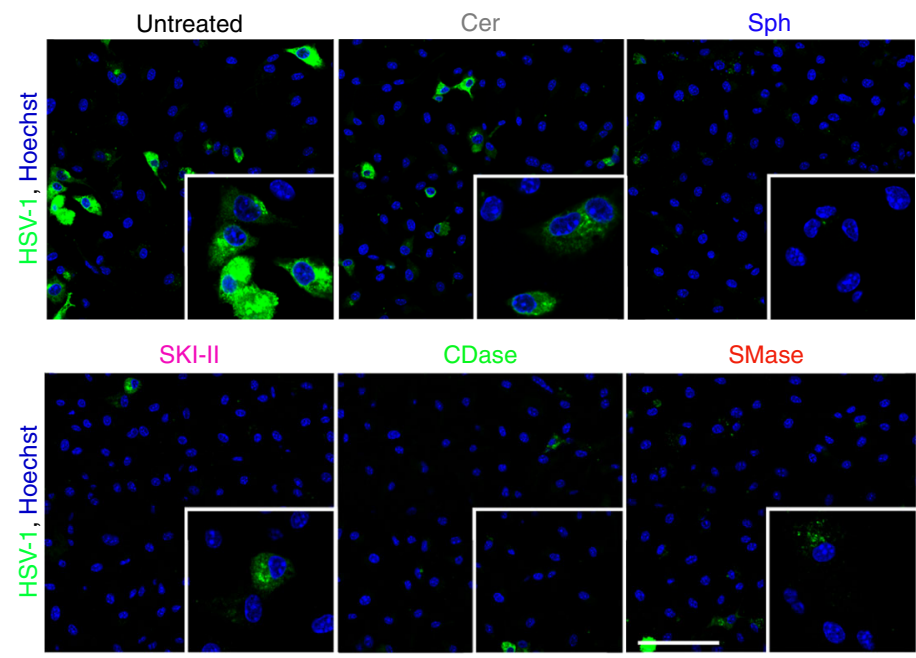

SMase
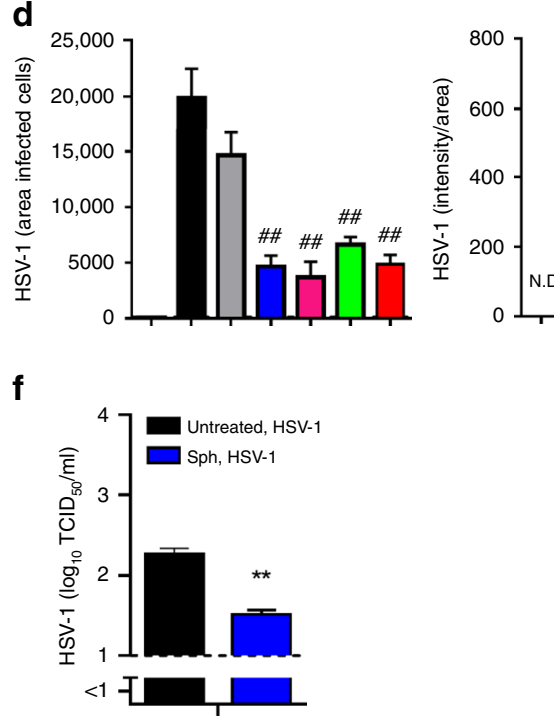

b

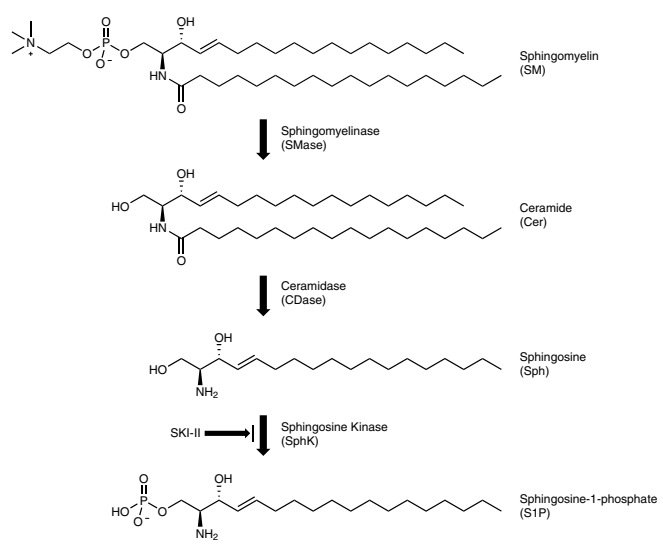

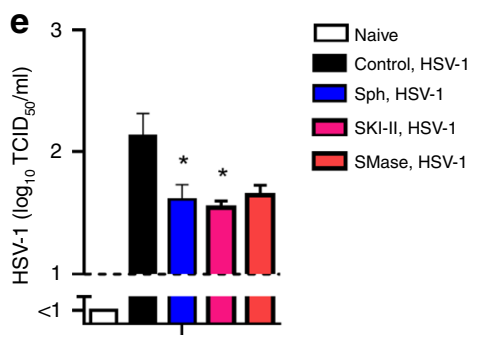

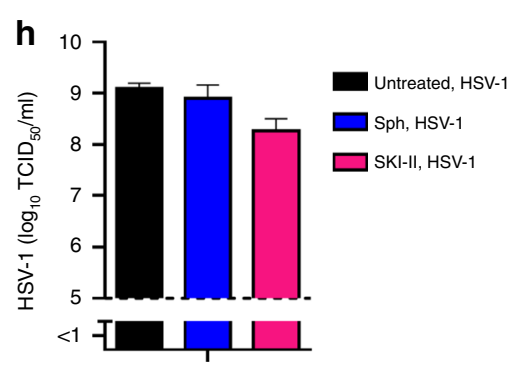

Fig. 4 Sphingosine protects against HSV-1 infection. a Immunofluorescence of wild-type (WT) macrophages, which were infected with HSV-1 (multiplicity of infection [MOI] 10) and stained for acid ceramidase (aCDase) and HSV-1 ( $n=5$, blue represents Hoechst staining, scale bar $10 \mu \mathrm{m}$ ) after 30 min. b Schematic representation showing the metabolism of sphingomyelin (SM) to sphingosine-1-phosphate (S1P). c, d Representative image (c) and quantification (d) of immunofluorescence from bone marrow derived macrophages (BMDMs) that have been incubated for 30 min with $\beta$-D-galactosyl ceramide (Cer; $100 \mu \mathrm{M})$, D-erythro-sphingosine (Sph; $100 \mu \mathrm{M})$, sphingosine kinase inhibitor (SKI-II; $100 \mu \mathrm{M})$, ceramidase (CDase; $250 \mathrm{U} / \mathrm{L})$ or sphingomyelinase (SMase; $6.5 \mathrm{U} / \mathrm{ml}$ ) and subsequently infected with HSV-1 at a multiplicity of infection (MOI) of 100 ( $n=5$, scale bar $50 \mu$ m, one-way ANOVA [Dunnett's multiple comparison]), fixed and stained after 6 h. N.D.: not done. e Tissue culture infection dose 50 (TCID 50 ) of BMDMs that have been incubated for 30 min with D-erythro-sphingosine (Sph; $40 \mu \mathrm{M}$ ), sphingosine kinase inhibitor (SKI-II; $200 \mu M$ ), or sphingomyelinase (SMase; $6.5 \mathrm{U} / \mathrm{ml}$ ), subsequently infected with HSV-1 at a MOI of 1 and analyzed after $24 \mathrm{~h}$ ( $n=3-4$, one-tailed student's $t$-test). f TCID 50 of THP-1 cells that have been incubated for $30 \mathrm{~min}$ with D-erythro-sphingosine (Sph; $400 \mu \mathrm{M})$ and subsequently infected with HSV-1 at a MOI of 0.01, analyzed after $6 \mathrm{~h}(n=3$, two-tailed student's t-test). $\mathbf{g} \mathrm{TCID}_{50}$ of HeLa cells that have been incubated for 30 min with D-erythro-sphingosine (Sph; $100 \mu \mathrm{M}$ ), and sphingosine kinase inhibitor (SKI-II; $100 \mu \mathrm{M})$, and subsequently infected with HSV-1 at a MOI of 0.001 analyzed after $24 \mathrm{~h}$ ( $n=3$, one-way ANOVA [Dunnett's multiple comparison]). $\mathbf{h} \mathrm{TCID}_{50}$ of Vero cells that have been incubated for $30 \mathrm{~min}$ with D-erythro-sphingosine (Sph; $100 \mu \mathrm{M}$ ), and sphingosine kinase inhibitor $(\mathrm{SKI}-\mathrm{II} ; 100 \mu \mathrm{M})$, and subsequently infected with HSV-1 at a MOI of 0.01 analyzed after $24 \mathrm{~h}(n=3$, two-tailed student's $t$-test). All data are shown as mean \pm SEM. ${ }^{\star} p \leq 0.05,{ }^{\star \star} p \leq 0.01,{ }^{*} p \leq 0.001,{ }^{*}{ }^{*} p \leq 0.0001$. 
a

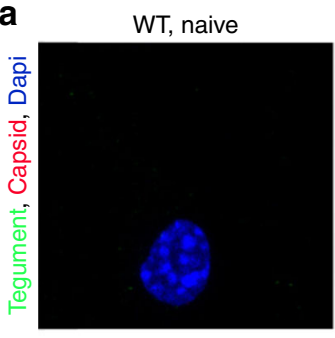

C
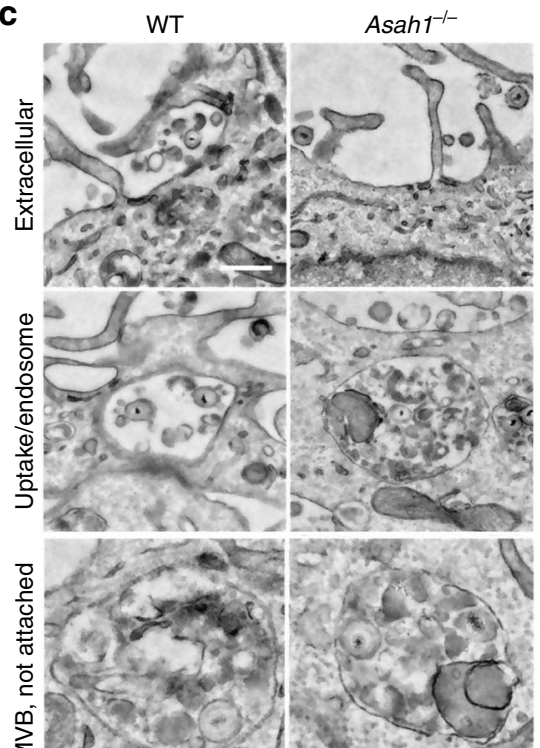

$\sum$

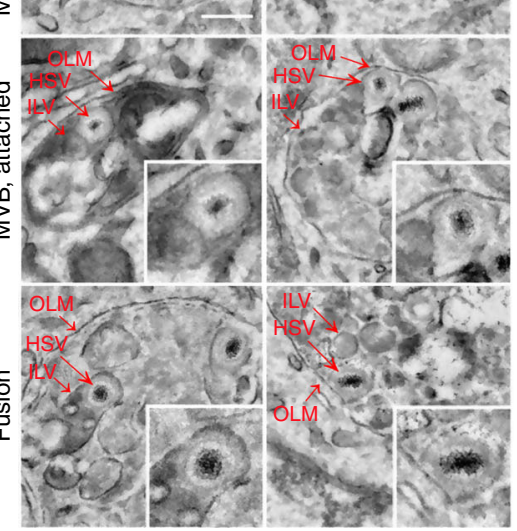

d

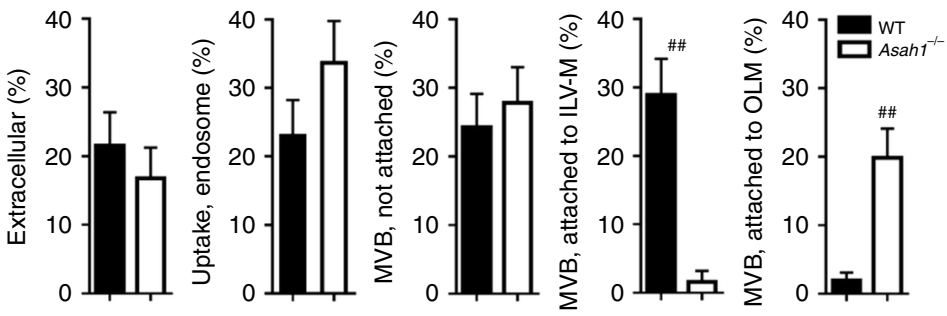

b

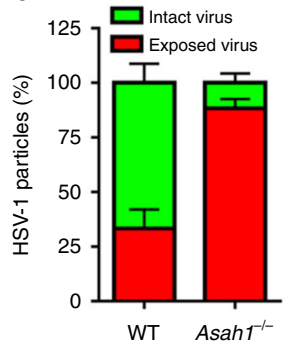

e
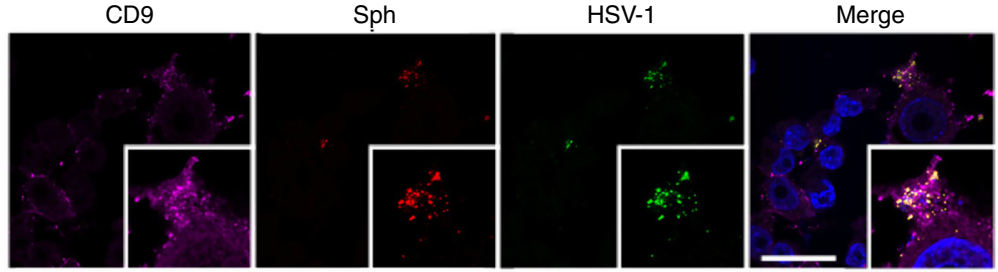

$f$
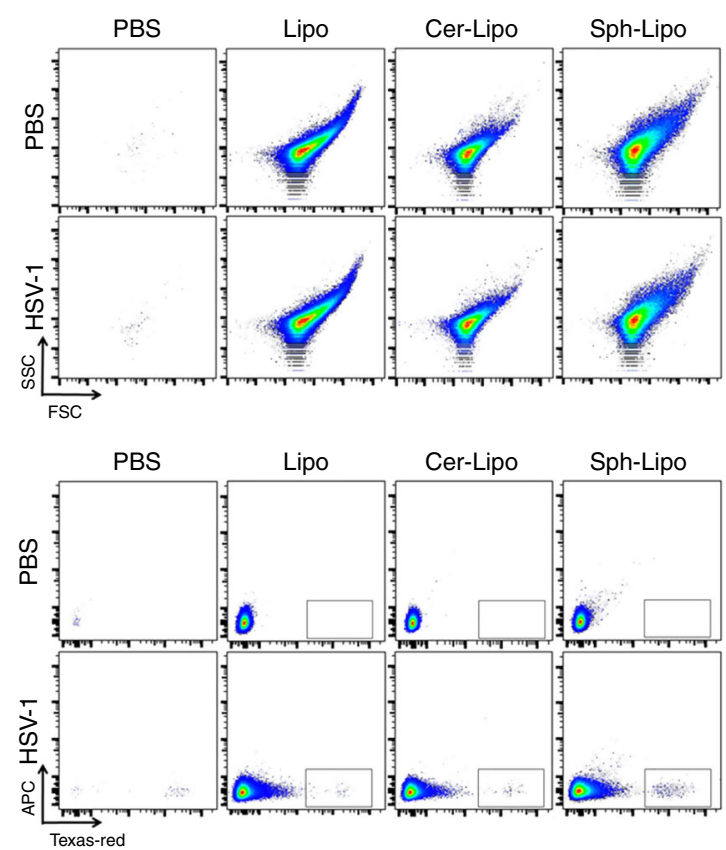

were not released from the envelope. In strong contrast, in aCDase lacking (Asah1-l-) macrophages we detected almost exclusively exposed capsids without tegument (Fig. 5a, b), suggesting that the capsids were released from the envelope and the tegument due to virus infection. To get more insights into the reason for limited infection in WT macrophages, we performed electron microscopy experiments in WT and Asah $1^{-1-}$ macrophages. WT and Asah1 $1^{-1-}$ macrophages showed similar amounts of HSV-1 particles attached to the plasma membrane (Fig. $5 \mathrm{c}, \mathrm{d}$ ). In line, similar amounts of HSV-1 particles were detected in WT and Asah1 $1^{-/-}$macrophages in endosomes and MVB (Fig. 5c, d). In WT macrophages HSV-1 particles were located in close 
Fig. 5 Sphingosine-rich intraluminal vesicles trap HSV-1. a, b Representative images (a) and quantification (b) of immunofluorescence from wild-type (WT) and Asah1-/- bone marrow derived macrophages (BMDMs) incubated with HSV-1-VP16-GFP (multiplicity of infection [MOI] 30) for 30 min. Intact viral particles are visible through GFP-tagged tegument protein VP16 (green). Exposed capsids were stained with anti-VP5 (red, $n=17$ images, one of two experiments is shown, scale bar $10 \mu \mathrm{m}$ ). c, d Representative electron microscopy images (c) and quantification (d) of WT and Asah1-/- BMDMs infected with HSV-1 (MOI 250) analyzed after $30 \mathrm{~min}\left(n=68\right.$ WT images and $n=63$ Asah $1^{-/}{ }^{-}$images from three independent experiments; upper scale bar $400 \mathrm{~nm}$, lower scale bar $200 \mathrm{~nm}$, Mann-Whitney test). e Immunofluorescence of WT BMDMs, which were loaded with clickable $\omega$-azido-sphingosine for $3 \mathrm{~h}$, infected with HSV-1 (MOI 10) for 30 min and then stained for sphingosine, the intraluminal vesicle (ILV) marker CD9, HSV-1 anti-capsid and Hoechst (blue, $n=5$, scale bar $20 \mu \mathrm{m}$ ). $\mathbf{f}, \mathbf{g}$ Representative dot plots $(\mathbf{f})$ and quantification ( $\mathbf{g}$ ) of control liposomes, ceramide-loaded liposomes and sphingosineloaded liposomes that were incubated with fluorescently labeled HSV-1 for 10 min and then analyzed for HSV-1 binding in flow cytometry ( $n=6$, 2-way ANOVA [Tukey's multiple comparison]). All data are shown as mean \pm SEM. ${ }^{\star} p \leq 0.05,{ }^{\star \star} p \leq 0.01,{ }^{*} p \leq 0.001,{ }^{\#} p \leq 0.0001$.

proximity to the ILVs, and in some cases appeared to fuse with ILVs (Fig. 5c, d). This correlated with trapping of HSV-1 in ILVs (Fig. 5c, d). In contrast, in Asah $1^{-1-}$ macrophages, the HSV-1 envelopes were more frequently associated with the outer limiting membranes (OLMs) of the MVBs (Fig. 5c, d), so that membrane fusion here led to a productive release of capsids into the cytosol and infection of the cell. Therefore, we concluded that aCDasederived sphingosine mediates binding of HSV-1 envelope to ILVs membrane, which results in trapping of the virus.

To prove that indeed sphingosine on ILVs interacted with HSV-1 we loaded WT macrophages with $\omega$-azido-sphingosine, which can be visualized by a click reaction ${ }^{42} .3 \mathrm{~h}$ after sphingosine loading, we infected cells with HSV-1 and co-stained cells with the ILV marker CD9. Indeed, sphingosine accumulated in ILVs (Fig. 5e). As expected HSV-1 co-stained with these sphingosinerich ILVs (Fig. 5e). To determine that indeed sphingosine is responsible for interaction between HSV-1 and ILVs, we generated liposomes and loaded them either with ceramide or sphingosine. Liposomes were then incubated with HSV-1 and binding of virions was analyzed after $10 \mathrm{~min}$ by flow cytometry. Control and ceramide-liposomes did not bind HSV-1 (Fig. 5f, g). In contrast, sphingosine-liposomes bound HSV-1 (Fig. 5f, g). Concluding, we found that the antiviral effects of aCDase are mediated via fusion of viral envelop to sphingosine containing ILVs of MVBs.

IRF8, but not IFN-I signaling, induces aCDase expression. Our data show that the Asah1 gene encoding aCDase is a crucial factor involved in antiviral protection against HSV-1. Next, we aimed to define, how aCDase expression is induced. We aimed to analyze whether Asah1 is also induced by IFN-I. The results show that Asah1 expression was hardly influenced by IFN-I, nor did Asah1 influence the induction of IFN-I or interferon induced genes (Fig. 6a, b). Therefore we concluded that Asah1 is not specifically upregulated during infection, but shows already basal activity in macrophages before infection. To get insights how Asah1 expression is upregulated in macrophages we considered that interferon regulatory factor 8 (IRF8) might play a role in this process. IRF8 is a factor, which induces natural resistance in macrophages during their development ${ }^{43,44}$. Furthermore, it was shown that IRF8 regulates the expression of aCDase during the induction of apoptosis in cancer cells ${ }^{45}$. RT-PCR analysis of myeloid pre-cursor cells in the bone marrow revealed IRF8dependent upregulation of Asah1 (Supplementary Fig. 5). To determine the role of IRF8 in regulating Asah1 in mature macrophages, we analyzed Asahl expression in the spleen, an organ showing high numbers of macrophages. RT-PCR and Western blot analysis revealed limited levels of Asah1 mRNA and aCDase precursor protein in spleens of $\operatorname{Irf} 8^{-1-}$ mice when compared to WT spleens (Fig. 6c, d, Supplementary Fig. 8). In line, Irf8 $8^{-1-}$ mice showed elevated levels of HSV-1 DNA as well as increased production of viral particles after infection with HSV-1 (Fig. 6e, f). It has previously been reported, that IRF8 expression in human blood monocytes decreases with age, which correlates with decreased expression of interferon stimulated genes and might be a reason for impaired antiviral resistance to influenza A virus (IAV) ${ }^{46}$. Whether this reduced IRF8 expression influences also aCDase dependent virus control remains to be studied. We concluded that Asah1 is not induced during infection or by IFN-I, however IRF8 regulates Asah1 expression during development of macrophages.

\section{Discussion}

Macrophages are one of the most important effector cells of the innate immune system ${ }^{5,47}$. During infection with viruses such as vesicular stomatitis virus (VSV), lymphocytic choriomeningitis virus (LCMV), adenovirus or mouse hepatitis virus, macrophages play a major protective role in limiting infection and preventing severe disease $\mathrm{e}^{2-4,48-50}$. In particular, tissue resident macrophages such as Kupffer cells in the liver clear a range of systemic virus infections ${ }^{4,48}$. Molecular mechanisms explaining this strong antiviral activity are presently unknown. Here we found that macrophages are essential for the control of HSV-1. With our in vitro data, we linked the strong antiviral activity of macrophages to the expression of aCDase. aCDase produced sphingosine-rich ILVs in MVBs. Once HSV-1 is taken up into MVBs, sphingosine-rich ILVs bind to HSV-1 particles and thereby trap them in MVBs and prevent infection of the cell. Expression of aCDase was not regulated by IFN-I, but was induced in macrophages in response to IRF8 signaling. While we focused in our manuscript on the role of aCDase in macrophages, our in vivo data suggest that aCDase exerts antiviral activity probably also in other myeloid or even none-myeloid cell types.

It remains to be explored whether the restriction mechanism of sphingosine-rich ILVs is specific for HSV-1 or whether also other viruses are sensitive to sphingosine-rich ILVs. Interestingly, VSV, the flaviviruses Japanese encephalitis virus (JEV) and yellow fever virus (YFV) fuse with ILVs in MVBs ${ }^{51,52}$. Le Blanc et al. ${ }^{51}$ and Nour et al. ${ }^{52}$ considered the release of viral capsids into ILVs a relevant step in virus life cycle, and suggested that back-fusion of ILVs, harboring capsids, with the OLMs of MVBs leads to productive infection. Our data show that although back-fusion might occur in macrophages, fusion of viral membrane with ILV membrane is rather a cellular mechanism to limit infection of the cell. Further experiments are required to determine whether other enveloped viruses and non-enveloped viruses are also restricted by sphingosine in MVBs.

Viruses frequently have an acidic isoelectric point at low $\mathrm{pH}^{53}$, implying that these viruses are negatively charged at physiological $\mathrm{pH}$. The isoelectric point of HSV-1 is around $4.9^{54}$. In contrast, sphingosine is net positively charged at neutral or acidic $\mathrm{pH}$, as occurring in MVBs. We suggest that sphingosine gives ILVs a positive charge, which facilitates the interaction with negatively charged HSV-1. Indeed it was shown that sphingosine containing vesicles interact with negatively charged membranes at the plasma membrane or in late endosomes and accelerates fusion of 

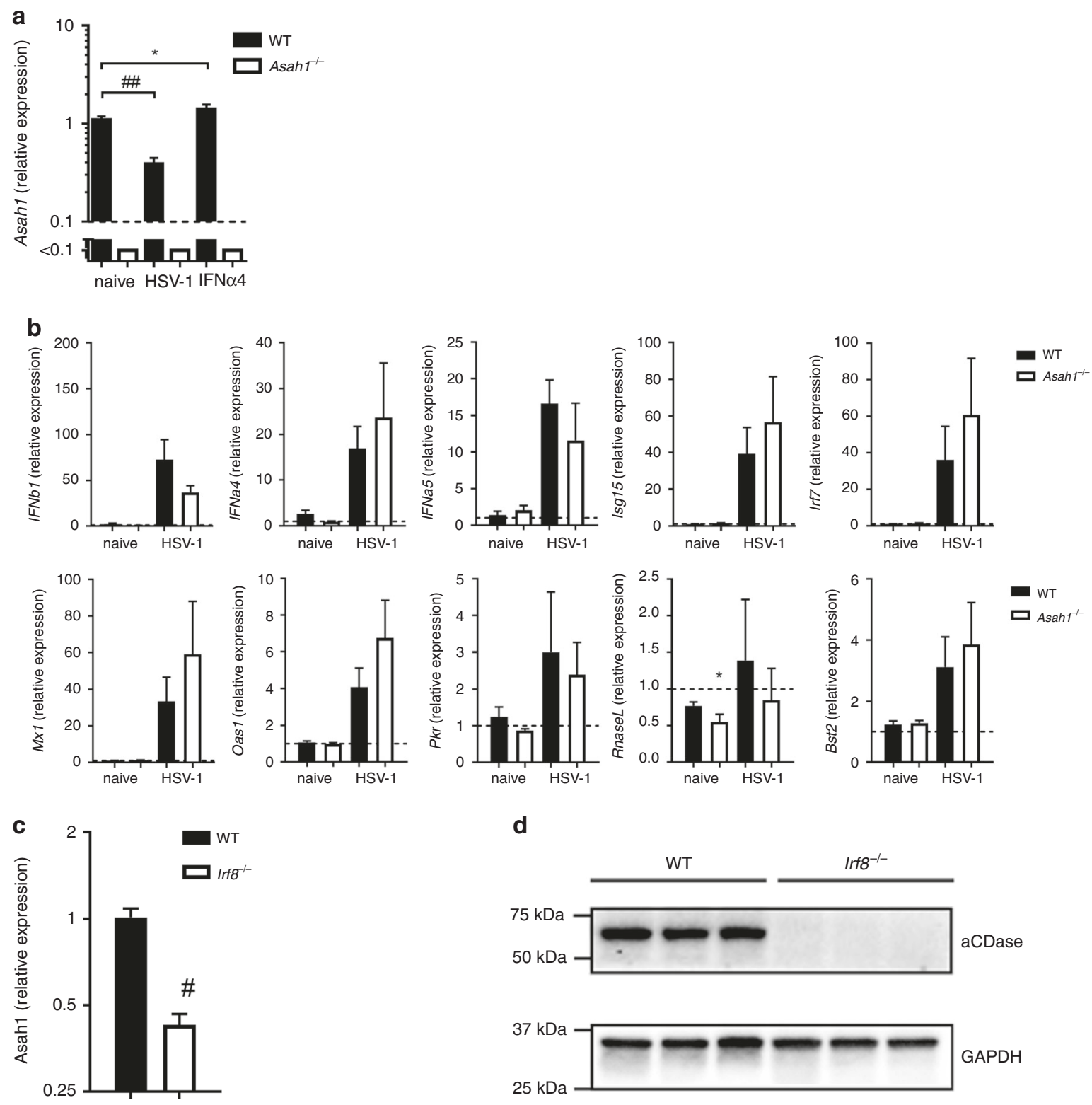

d

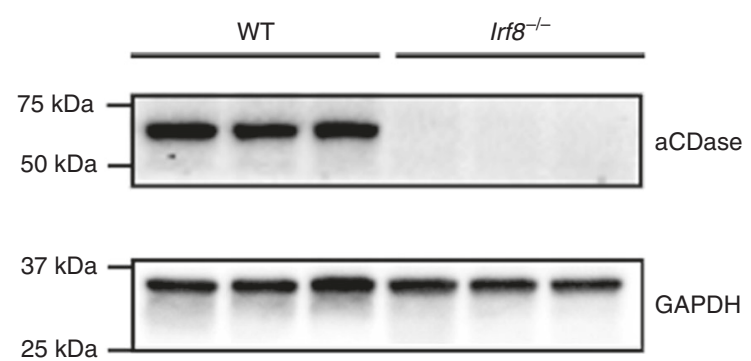

e

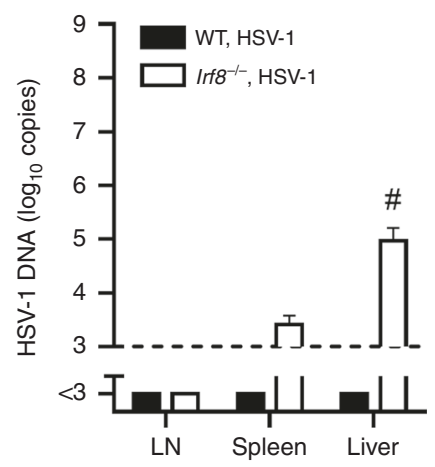

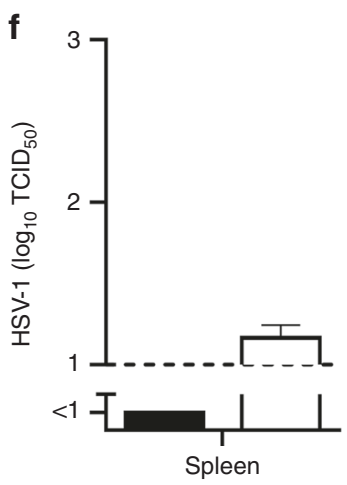

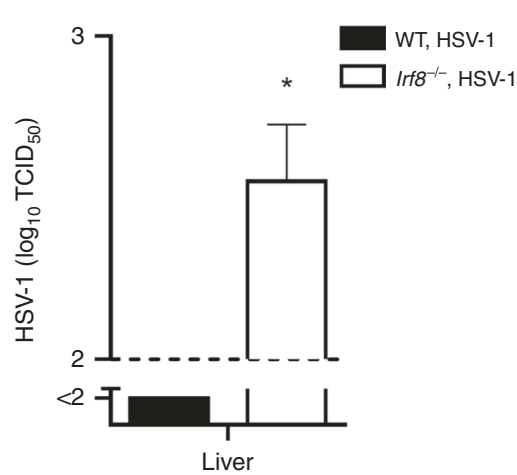

Fig. 6 IRF8, but not IFN-I signaling, induces aCDase expression. a Real-time polymerase chain reaction (RT-PCR) of wild-type (WT) and Asah1-/- bone marrow derived macrophages (BMDMs) that were left untreated, infected with herpes simplex virus type 1 (HSV-1) at a multiplicity of infection (MOI) of 1 for $24 \mathrm{~h}$ or infected and treated with 100 units interferon- $\alpha 4$ (IFN- $\alpha 4 ; n=10$, one-way ANOVA [Dunnett's multiple comparison]). b RT-PCR of WT and Asah1-/- BMDMs that were left untreated or infected with HSV-1 at an MOI of 1 for $24 \mathrm{~h}$ ( $n=10$, two-tailed student's $t$-test). $\mathbf{c}, \mathbf{d}$ RT-PCR for Asah1 (c, $n=$ 5 , two-tailed student's $t$-test) and Western blot for aCDase $(\mathbf{d}, n=3)$ of spleen tissues from WT and interferon regulatory factor 8 deficient (Irf8-/-) mice that were left untreated. e, $\mathbf{f}$ RT-PCR (e) of lymph nodes (LN), spleens and livers and tissue culture infection dose 50 (TCID 50 , f) of spleens and livers from WT and Irf8- $/-$ mice that were infected with $2 \times 10^{6}$ TCID $_{50}$ HSV-1 and analyzed on day $3(n=5-6$, two-tailed student's $t$-test). All data are shown as mean \pm SEM. ${ }^{\star} p \leq 0.05,{ }^{* *} p \leq 0.01,{ }^{\#} p \leq 0.001,{ }^{\# \#} p \leq 0.0001$. 
these membranes ${ }^{55,56}$. More biophysical work is required to better understand the interaction of HSV-1 with sphingosine.

We compared the in vivo phenotype of $A s a h 1^{-I-}$ mice during HSV-1 infection to several other mouse strains, which show

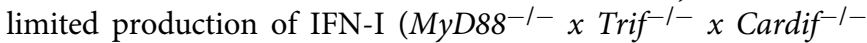
mice), limited response to IFN-I (Ifnar ${ }^{-/}$mice) or lack SAMHD1 (Samhd1 ${ }^{-1-}$ mice). SAMHD1 was previously reported to be of importance for HSV-1 control in myeloid cells ${ }^{36,57}$ and anti-viral effects can be evaded by some herpesviruses ${ }^{58}$. Viral replication in $A s a h 1^{-1-}$ was comparable to $M y D 88^{-1-} \times$ Trif $^{-1-}$ $x$ Cardif $^{-1-}$ mice and Ifnar ${ }^{-1-}$ mice and even enhanced to that in Samhd $1^{-1-}$ mice. These findings indicate that Asah1 is an important and major innate effector mechanism. We observed increased HSV-1 DNA in livers of Samhd1 $1^{-1-}$ mice. This is in line with other groups which determined an important role of SAMHD1 on HSV-1 control in vitro ${ }^{36,57,58}$. How this lack of HSV-1 control influences disease onset after HSV-1 infection needs to be determined in the future. While SAMHD1 and IFN signaling counteract mainly viral replication and production of infectious particles, we think that aCDase protects macrophages from infection with HSV-1 immediately after the virus is taken up.

Our electron microscopy studies indicate that HSV-1 fuses with ILVs. We consider that trapped viral particles are unable to leave MVBs and are targeted to lysosomes for degradation. This pathway of degradation might also facilitate antigen-presentation and immune activation, as high numbers of tetraspanins and MHC-II molecules are contained within ILVs ${ }^{23,59}$. More studies are required to identify the relationship of aCDase, sphingosine and antigen-presentation on MHC-II. In our study we focused mainly on the role of sphingosine and aCDase in macrophages. In addition, aCDase is expressed in several other tissues, especially in lung, colon and stomach as well as spleen ${ }^{60}$ (Supplementary Fig. 6). Cells in these organs are involved in building a barrier, which protects against invading viruses. We consider it likely that in addition to macrophages, potentially also other cell types express aCDase in MVBs and are thereby protected against invading viruses.

From our data we suggest enhanced virus infections in patients with Farber disease, which are deficient in activity of aCDase ${ }^{61}$. While such associations were not determined yet, patients with Farber disease have a limited life span and die early after birth ${ }^{61}$. Akin to other innate or adaptive deficiencies, a limited lifespan of the patients may obscure a link between innate or adaptive deficiencies to infectious agents ${ }^{62}$. Whether other mutations, which affect the activity of aCDase, are widespread in the population and whether such mutations influence the susceptibility to viral infections will be subject to future studies.

In conclusion, we found that macrophages are essential to survive an infection with HSV-1. Mechanistically, macrophages took up HSV-1 and accumulated it in MVBs. aCDase in macrophages enriched ILVs with sphingosine. Once HSV-1 entered MVBs sphingosine-rich ILVs trapped HSV-1 and prevented productive infection of the cells.

\footnotetext{
Methods

Mice. For acid ceramidase 1 (Asahl) deficient heterozygous mice, the exon-intron organization of the gene was established on the basis of the Asah1 cDNA sequence. A targeting vector was constructed, including Asah1 genomic sequences on the $\mathrm{C} 57 \mathrm{BL} / 6$ genetic background, a long homology region of $5.8 \mathrm{~kb}$ and a short homology region of $1.7 \mathrm{~kb}$. It also included two loxP sites flanking Asah1 exon 1, a neomycin gene flanked by flipase recognition target sites for positive selection of homologous recombination, and a diphtheria toxin, a negative-selection marker to detect insertions and nonhomologous recombinant embryonic stem cell clones. This targeting vector was inserted into C57BL/6 embryonic stem cells by homologous recombination. Offspring were crossed with CreER mice (Gt[ROSA]

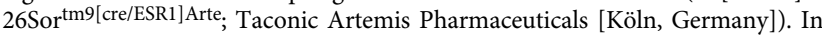
some experiments we used mice in which acid ceramidase deficiency could be induced $\left(\right.$ Cre $\left.\times A s a h 1^{f l / f l}\right)$. CreER x Asah $1^{f l f l}$ offspring express a Cre recombinase under the tamoxifen promoter and the Asah1 gene with two LoxP sites flanking exon 1. Administering tamoxifen thus led to the expression of Cre recombinase and loss of function mutation by deletion of exon 1 in the Asahl gene. In this model, knock out of Asah1 occurs right before infection. Potential developmental defects due to long term knock out of Asah1 are excluded in this model.

Asah $1^{f l f l} \times$ EIIa-Cre mice carry a Cre transgene under the control of the adenovirus EIIa promoter that targets expression of Cre recombinase to the early mouse embryo, and are used for germ line deletion of Asah1 gene, referred to

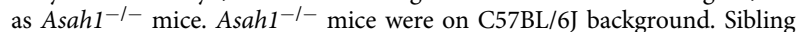
animals with the genotype Asah1 $1^{+/+}$were used as control animals. We bred

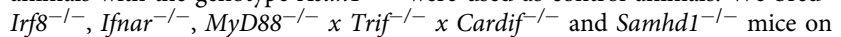
C57BL/6J background. Samhd1 $1^{-1-}$ mice were originally developed by Prof. Jan Rehwinkel (MRC Human Immunology Unit, MRC Weatherall Institute of Molecular Medicine, University of Oxford, Oxford, England $)^{63}$.

All animals were housed in single ventilated cages. Animal experiments were authorized by the Landesamt für Natur, Umwelt und Verbraucherschutz (LANUV) Nordrhein-Westfalen and in accordance with the German law for animal protection and/or according to institutional guidelines at the Ontario Cancer Institute of the University Health Network.

Cell lines, bone marrow macrophages. Raw264.7 cells were purchased from the American Type Culture Collection (ATCC, Manassas, VA, USA, \#: ATCC: TIB71). THP-1 cells were purchased from the German Collection of Microorganisms and Cell Cultures (DSMZ, Braunschweig, Germany, \#: DSMZ: ACC 16). Vero cells were provided by the Ontario Cancer Institute (Toronto, ON, Canada). HeLa cells were provided in house (University Clinic Essen, Essen, Germany). For the generation of macrophages, bone marrow was isolated from mice and cultured for 9 days with macrophage colony-stimulating factor (M-CSF). Cells were plated on new cell culture plates and the experiment was started the next day. For generation of primary fibroblasts, lungs were isolated from mice and digested using DNase/ Liberase. Fibroblasts were harvested after 6 days of culture and experiment was started the subsequent day.

Viruses. We used HSV-1(17+)Lox and HSV-1(17+)Lox-CheVP26 in which monomeric Cherry has been fused to the $\mathrm{N}$-terminus of the small capsid protein VP26 ${ }^{64}$, HSV-1F (kindly provided by Prof. Hartmut Hengel, Institute of Virology, Freiburg, Germany) and HSV-VP16-GFP65 (kindly provided by Dr. Yohei Yamauchi, University of Bristol, United Kingdom)

Reagents. D-erythro-sphingosine $(\mathrm{C} 18,860490)$ and D-Galactosyl- $\beta 1-1$ '-N-Nervonoyl-D-erythro-sphingosine (C24:1, $\beta$-D-Galactosyl Ceramide, 110759), were obtained from Avanti Polar Lipids (Alabaster, AL, USA). Glycoceramidase (E9030100MUN), sphingomyelinase (S8633-25UN), Cy3-linked DBCO (777366-1 mg) and DAPI (D9542) were purchased from Millipore Sigma (Darmstadt, Germany). Recombinant mouse Asah2 (3558-AH) was purchased from R\&D Systems (Minneapolis, MN, USA). The sphingosine kinase inhibitor SKI-II (CAS \# 312636-16-1; Cat \# 2097) was obtained from Tocris Bioscience (Bristol, United Kingdom) and tamoxifen (CAS \# 10540-29-1, Cat \# T5648-5G) and cornoil (CAS \# 8001-30-7, Cat \# C8267-500ML) from Millipore Sigma. Clodronate and control liposomes were purchased from Liposoma (CP-005-005, Amsterdam, Netherlands). Clickable $\omega$ azido-sphingosine ( $(2 S, 3 R, E)$-2-amino-18-azidooctadec-4-ene-1,3-diol) for click chemistry $^{42}$ staining was synthesized in-house by Julian Fink.

Tamoxifen treatment. Tamoxifen was dissolved in corn oil. Eight, six, and four days before an experiment, $\mathrm{Cre}^{-} \times A s a h 1^{f l / f l}$ control animals and $\mathrm{Cre}^{+} \times A s a h 1^{f l / f l}$ animals were treated with $4 \mathrm{mg}$ tamoxifen (in $100 \mu \mathrm{L}$ corn oil) intraperitoneally.

Depletion of macrophages. For macrophage-depleted mouse experiments, mice were treated intravenously with $50 \mathrm{mg} / \mathrm{kg}$ clodronate to deplete tissue resident macrophages. After 3 days, mice were ready to be used in experiments.

Survival experiments. To analyze the effect of macrophage/ sphingosine deficiency on the course of infection, we performed survival experiments. For this purpose, animals were infected with a dose that was sublethal for WT animals. Animals were checked daily, killed with corresponding termination criteria, and counted as dead. Termination criteria were body weight, general condition, spontaneous behavior and clinical findings.

Bone marrow chimera mice. For bone marrow chimera experiments, 10-12 weeks old female C57BL/6J mice were irradiated with $9.5 \mathrm{~Gy}$. On the following day, bone marrow from donor mice was isolated under sterile conditions and i.v. administered. On day 10 after irradiation, mice were treated with clodronate liposomes to deplete tissue resident macrophages. After 50 days of reconstitution mice were ready to be used in experiments. 
Infection of cells and animals. For in vitro infection, the virus was added to the cell cultures. After incubation for $1 \mathrm{~h}$, the supernatant was washed away and new medium was added. The supernatant was collected at various times after infection. For intravenous infection, $100 \mu \mathrm{L}$ of the virus was injected into the tail vein. For intravaginal infection, $20 \mu \mathrm{L}$ of the virus was pipetted into the vagina of anesthetized mice. The vagina was briefly closed with skin glue to reduce variability of inoculation time and volume.

Immunofluorescence microscopy. Immunofluorescence was used to detect viruses in cells and organs. For in vitro experiments, cells were seeded in 24-well plates, each containing a coverslip. After $24 \mathrm{~h}$, the cells were correspondingly infected, fixed after incubation, and labeled with antibodies. Organs of infected animals were snap-frozen, and $8 \mu \mathrm{m}$ thick sections were recovered on a microscope slide. The sections were fixed, and virus was visualized with virus-specific antibodies. The following primary antibodies were used: anti-HSV-1 capsid (SY4563 34 , anti-capsid, 1:100), anti-HSV-1 glycoprotein (hu2 $\mathrm{c}^{66}$, anti-glycoprotein $\mathrm{gB}, 1: 200$ ) or anti-HSV-1 VP5 (NC-1 ${ }^{41}$, anti-capsid, 1:500). Alternatively, virus was directly labeled with a dye (e.g., Cy3; carboxylfluorescein succinimidyl ester [CFSE]) was used. Zeiss ELYRA PS.1 SIM/PAL-M/STORM/TIRF and LSM710 (ZEISS, Oberkochen, Germany), was used to acquire images.

Immunofluorescence microscopy of sphingosine. Immunofluorescence was used to detect sphingosine in cells. Cells were seeded in 24 -well plates, each containing a coverslip. After $24 \mathrm{~h}$, the cells were correspondingly treated, fixed after incubation, and labeled with antibodies. As primary antibody monoclonal anti-sphingosine antibody (clone NHSPH, ALF-274042010, Alfresa Pharma Corporation, Osaka, Japan, 1:1000) and as secondary antibody Cy3-coupled anti-IgM F(ab)2 fragments (Jackson ImmunoResearch, Ely, United Kingdom, 1:500) were used. Samples were examined with a Leica TCS-SP5 confocal microscope equipped with a $100 \times$ lens, and images were analyzed with Leica LCS software version 2.61 (Leica Microsystems, Mannheim, Germany). Fluorescence intensity was determined in 100 randomly selected cells/sample (total of 300 cells). We determined the fluorescence of the cell periphery.

\section{Detection of HSV in cell supernatant/organs by TCID $_{50}$ assay. For tissue} culture infection dose $50\left(\mathrm{TCID}_{50}\right)$ Vero cells were seeded onto a 96-well plate and let grow to confluence overnight. To determine the viral titer, cell culture supernatant or organ suspension was titrated and transferred to the 96-well plate. After 7 days of incubation, the plaque positive wells were counted, and the number of infectious particles per $\mathrm{ml}$ of supernatant or organ was determined.

Detection of HSV in organs by qRT-PCR. For quantitative real-time polymerase chain reaction (qRT-PCR) DNA was isolated from organs using the innuPREP Virus DNA Kit (Order \#: 845-KS-4600050, Analytik Jena, Jena, Germany). RealStar HSV PCR Kit 1.0 CE (Order \#: 061013, Altona Diagnostics, Hamburg, Germany) was used to quantify the amount of HSV-1 genomes per organ. A LightCycler 480 Instrument (Roche Life Science, Basel, Switzerland) was used for detection.

Capsid uncoating assay of herpes simplex virus. Cells were incubated with HSV-1 expressing GFP at the tegument protein VP16 (HSV-1-VP16-GFP65). After 30 min of incubation, cells were fixed and stained for HSV capsid with anti-VP5 $\left(\mathrm{NC}-1^{41}, 1: 500\right)$ and DAPI. Images were acquired on Leica SP8 microscope (Wetzlar, Germany) and analyzed using CellProfiler by segmentation and counting of single VP16-GFP or VP5 puncta.

Sphingosine-containing liposomes for FACS analysis. Liposomes were prepared by sonication as previously described ${ }^{67}$. Phosphatidylcholine (PC), cholesterol (Ch), sphingomyelin (SM), ceramide (Cer) and D-sphingosine (Sph) were from Millipore Sigma. The lipid composition of the liposomes was as follows: controlliposomes (PC: Ch: SM 33.3:33.3:33.3 mol/\%), ceramide-rich liposomes (PC: Ch: SM: Cer 33:33:24:10 mol/\%) and sphingosine-rich liposomes (PC: Ch: SM: Sph 33:33:24:10 mol/\%).

Isolation of total RNA, reverse transcription and qRT-PCR. Total RNA was isolated from cells using Trizol. Complementary DNA synthesis was performed with the QuantiTect Reverse Transcription Kit (Qiagen, Hilden, Germany).

For quantitative real-time polymerase chain reaction (qRT-PCR) either Fast SYBR Green Master Mix (Applied Biosystems, Darmstadt, Germany) or TaqMan Fast Universal PCR Master Mix (ThermoFisher Scientific) was used on the 7500 Fast Real-Time PCR System (Applied Biosystems). For analysis, the expression levels of all target genes were normalized against glyceraldehyde 3-phosphate dehydrogenase (Gapdh; $\Delta \mathrm{Ct}$ ). Relative gene expression values were calculated with the $\Delta \Delta \mathrm{Ct}$ method. Following primers were used: Gapdh (Mm99999915_g1, ThermoFisher Scientific), Gapdh (QT01658692, Qiagen), Asah1 (Mm00480021 m1, ThermoFisher Scientific) IRF7 (Mm00516788_m1, ThermoFisher Scientific), Isg15 (QT00322749, Qiagen), RnaseL (QT01066086, Qiagen), Tetherin/Bst2 (QT01066184,Qiagen), IFNa4 (QT01774353, Qiagen),
IFNa5 (QT00327656, Qiagen), IFNß1 (QT00249662, Qiagen), Mx-1 (QT01064231, Qiagen), Oas1 (QT01056048, Qiagen) and PKR (QT00162715, Qiagen).

Click chemistry. Clickable $\omega$-azido-sphingosine $((2 S, 3 R, E)$-2-amino-18-azidooctadec-4-ene-1,3-diol) was used for click chemistry reaction as described previously ${ }^{42,68}$. Briefly, cells were seeded and grown to confluence. $\omega$-azidosphingosine was added to cell culture medium $(100 \mu \mathrm{mol} / \mathrm{L})$ for $3 \mathrm{~h}$. Cells were washed with PBS and then Cy3-linked DBCO $(8 \mu \mathrm{g} / \mathrm{ml}$ diluted in PBS) was added for $7 \mathrm{~min}$ at room temperature. Samples were washed $3 \mathrm{x}$ with PBS and medium was added. Cell were further analyzed as described.

For detailed synthesis and analysis of $\omega$-azido-sphingosine see methodical information "chemical synthesis of $\omega$-azido-sphingosine" in Supplementary Information and Supplementary Figs. 9-20.

Microarray. Total RNA preparations were checked for RNA integrity with an Agilent 2100 Bioanalyzer. All samples in this study exhibited high-quality RNA Integrity Numbers (RIN 9.8-10). Synthesis of cDNA and subsequent fluorescence labeling of cRNA were performed according to the manufacturer's protocols (OneColor Microarray-Based Gene Expression Analysis/Low Input Quick Amp Labeling; Agilent Technologies, Waldbronn, Germany). Briefly, $100 \mathrm{ng}$ of total RNA was converted to cDNA, followed by in vitro transcription and incorporation of $\mathrm{Cy} 3$ CTP into nascent cRNA. After fragmentation, labeled cRNA was hybridized to AgilentSurePrint G3 Mouse GE $8 \times 60 \mathrm{k}$ microarrays for $17 \mathrm{~h}$ at $65^{\circ} \mathrm{C}$ and scanned as described in the manufacturer's protocol. Signal intensities on 20-bit TIFF images were calculated by Feature Extraction software (FE, Vers. 10.7.1.1; Agilent Technologies). Data analyses were conducted with GeneSpring GX software (Vers. 10.5; Agilent Technologies). Probe signal intensities were quantile-normalized across all samples for reduction of inter-array variability. Input data preprocessing was concluded by baseline transformation to the median of all samples.

To ensure innate immune activation macrophages and fibroblasts were infected with LCMV and treated with IFN- $\alpha 4$. To analyze the microarray data a gene set enrichment analysis with GSEA (Broad Institute, v3.069) using the Gene Ontology Cellular Components (CC) gene set database (c5.cc.v6.2 ${ }^{70}$, accessed on 15.07.19) was performed using default settings. Results were further subjected to network analysis using the Enrichment Map plugin v3.2.0 in Cytoscape v3.7.1 with the following settings: FDR q-value cutoff 0.05 , p-value cutoff 1, Metric: Jaccard + Overlap Combined (50/50) with cutoff 0.375 . Clusters were identified by the AutoAnnotate plugin v1.3 and labeled manually for visualization. For visualization gene sets upregulated in LCMV-infected and interferon-treated macrophages or fibroblasts were sorted to the left or right side of the diagram respectively. Several membrane modulating proteins from the families of sphingolipids (including gangliosides), cholesterol and phosphatidylcholine were considered for simplified comparison of macrophages and fibroblasts (see Supplementary Table 2).

Virus binding to liposomes. Liposomes were incubated with fluorescently labeled virus. Samples were diluted and further analyzed in flow cytometry (LSR Fortessa) Liposomes were detected by size and granularity. Frequency of liposomes, which bound the virus was determined by fluorescence according to the gating shown in Fig. 5f. Data was analyzed using the software FlowJo (FlowJo LLC).

FACS Sorting. Bone marrow was isolated from tibia and femur of mice and erythrocytes were lysed. To sort pre-progenitor cells staining for linage markers anti-CD3e (Cat \# 45-0031-80, eBioscience, 1:50), anti-GR1 (Cat \# 108428, BioLegend, 1:400), anti-Mac-1 (Cat \# 101228, BioLegend, 1:400), anti-CD4 (Cat \# 101228, BioLegend, 1:200), anti-CD8a (Cat \# 100734, BioLegend, 1:100), antiTCRb (Cat \# 109228, BioLegend, 1:100), anti-NK1.1 (Cat \# 45-5941-82, eBioscience, 1:100), anti-B220 (Cat \# 103236, BioLegend, 1:100) and anti-CD19 (Cat \# 115534, BioLegend, 1:200) was performed. Additionally anti-CD16/32 (Cat \# 101305, BioLegend, 1:200), anti-CD41 (Cat \# 133906, BioLegend, 1:100), antiSca-1 (Cat \# 108114, BioLegend, 1:200), anti-c-kit (Cat \# 47-1171-82, Invitrogen, 1:50), anti-CD105 (Cat \# 120412, BioLegend, 1:50) and anti-CD150 (Cat \# 115910 BioLegend, 1:400) were stained to further classify cells. To sort immature granulocytes, mature granulocytes and monocytes anti-Ly6G/Ly6C (Cat \# 17-5931-82, Invitrogen, 1:100), anti-CD11b (Cat \# 25-0112-82, eBioscience, 1:100) and antiCD115 (Cat \# 11-1031-85, eBioscience, 1:100) were used. Gating strategy was performed as shown in Supplementary Fig 5.

Western blot for aCDase protein expression. Frozen mouse spleen or cultured primary mouse cells were lysed in radioimmunoprecipitation buffer containing $1 \mathrm{x}$ HALT Protease Inhibitor Cocktail and $50 \mathrm{mM}$ EDTA (Thermo Fisher, Cat. no. 78430 ) using $100 \mu \mathrm{l}$ lysis buffer per $10 \mathrm{mg}$ organ or $30 \mu \mathrm{l}$ lysis buffer per $10^{6}$ cells respectively. Spleens were homogenized using a homogenizer (Qiagen Tissue Lyser II, Cat. no. 85300) for $2 \mathrm{~min}$ at $20 \mathrm{~Hz}$. Lysates were then centrifuged for $20 \mathrm{~min}$ at $20,000 \mathrm{x} \mathrm{g}$ at $4{ }^{\circ} \mathrm{C}$, keeping the supernatants. Protein concentration was measured using detergent compatible (DC) Protein Assay (Bio-Rad, Cat. no. 5000112), standardized to $4 \mu \mathrm{g} / \mu \mathrm{l}$ and prepared for SDS-PAGE by addition of $4 \mathrm{x}$ Laemmli sample buffer (Bio-Rad, Cat. no. 1610747), incubation at $95^{\circ} \mathrm{C}$ for $5 \mathrm{~min}$ followed by cool-down on ice. $30 \mu \mathrm{g}$ per organ or $10 \mu \mathrm{g}$ per cell lysate was loaded per lane into a precast TGX AnyKD Stain-free gel (Bio-Rad, Cat. no. 4568126), using 
Bio-Rad AllBlue protein standard (Cat. no. 1610373) and ran for $45 \mathrm{~min}$ at $140 \mathrm{~V}$ in Tris/glycine/SDS running buffer (Bio-Rad, Cat. no. 1610732). Transfer was performed using the Trans-Blot Turbo system (Bio-Rad, Cat. no. 1704150) with Mini PVDF Transfer Packs (Bio-Rad, Cat. no. 1704156). Membranes were blocked in Pierce StartingBlock (TBS) Blocking Buffer (Thermo Fisher, Cat. no. 37542) for $2 \mathrm{~h}$ and primary antibody was incubated shaking at $4{ }^{\circ} \mathrm{C}$ for 2 days: $1: 500$ for antiaCDase rabbit polyclonal (Proteintech, Cat. no. 11274-1-AP) for spleens, or 1:500 anti-aCDase rabbit polyclonal (ProSci, Cat. no. 4741) for cell lysates, and 1:1000 for anti-GAPDH mouse (Meridian Life Science, Cat. no. H86504M) diluted in blocking buffer. Membranes were washed three times with Tris-buffered saline- 0.1 $\%$ Tween 20 (TBS-T) and incubated with secondary antibody at 1:5000 (from GE Life Sciences, anti-Rabbit IgG, HRP-linked, Cat. no. NA934 or anti-mouse IgG, HRP linked, Cat no. NA931) diluted in $25 \%$ blocking buffer in TBS-T shaking at room temperature for $2 \mathrm{~h}$. After washing twice in TBS-T and twice in TBS membranes were incubated with Pierce ECL (Thermo Fisher, Cat. no. 32106) for 5 min and imaged on a Bio-Rad ChemiDoc MP (Cat. no. 1708280) with ImageLab version 6.0.1 (Bio-Rad)

Histology. Histological analysis of snap-frozen liver and spleen tissues or cell cultures was performed with monoclonal fluorescence labeled antibodies against HSV-1-capsid (SY4563, 1:100) anti-HSV-1 (hu2c ${ }^{66}$, anti-gB, 1:200), anti-F4/80 (12-4801-82, eBioscience, San Diego, CA, USA, 1:100), anti-Asah1 (4741, ProSci, San Diego, CA, USA, 1:200), anti-CD9 (124809, Biolegend, San Diego, CA, USA, 1:100) and anti-sphingosine (clone NHSPH, ALF-274042010, Alfresa Pharma Corporation, Osaka, Japan, 1:1000). Nuclei were stained using Hoechst 33342 (Cat \# B2261-100MG, Sigma, 1:5000). Zeiss ELYRA PS.1 SIM/PAL-M/STORM/TIRF and LSM710, Leica SP8 gSTED and FLIM, Leica TCS-SP5, or Keyence BZ-9000 (Keyence, Osaka, Japan) were used to acquire images. Quantification of histology was done using standard procedures in Fiji software version 1.52e (Image J, NIH, Bethesda, MD, USA) or Cell Profiler ${ }^{71}$. In Fiji we used the raw images, applied a mean filter of two/ten to exclude noise. We took the total signal area and the intensity per area after an empirically fixed threshold for further evaluation.

Mass spectrometry analysis. $10^{6}$ cells were subjected to lipid extraction with $1.5 \mathrm{~mL}$ methanol/chloroform $(2: 1, v: v)^{72}$. The extraction solvent contained $\mathrm{d}_{7}$-sphingosine ( $\mathrm{d}_{7}$-Sph), C17-ceramide, and C16- $\mathrm{d}_{31}$-sphingomyelin (all from Avanti Polar Lipids) as internal standards. Sample analysis was carried out by liquid chromatography tandem-mass spectrometry (LC-MS/MS) using either a TQ 6490 mass spectrometer (for Sph) or a QTOF 6530 mass spectrometer (for ceramides and sphingomyelins) (both from Agilent Technologies, Waldbronn, Germany) operating in the positive electrospray ionization mode (ESI+). The following selected reaction monitoring (SRM) transitions were used for quantification: $\mathrm{m} / z 300.3 \rightarrow 282.3$ for Sph and $\mathrm{m} / z$ $307.3 \rightarrow 289.3$ for $d_{7}-S p h$. The precursor ions of ceramide or sphingomyelin species (differing in the lengths of their fatty acid chains) were cleaved into the fragment ions $\mathrm{m} / \mathrm{z} 264.270$ (for ceramide) or $\mathrm{m} / \mathrm{z} 184.074$ (for sphingomyelin), respectively ${ }^{73}$. Quantification was performed with Mass Hunter Software (Agilent Technologies).

Electron microscopy. The samples were fixed with glutaraldehyde $(2.5 \%$ in $100 \mathrm{mM}$ phosphate buffer). Cells were then contrasted with $1 \%$ osmium tetroxide, $1 \%$ thiocarbohydrazide, $1.5 \%$ potassium ferrocyanide, and $1 \%$ uranyl acetate in water and were then dehydrated in an ascending ethanol row $(50 \%, 70 \%, 96 \%$, and $3 \times 100 \%, 10 \mathrm{~min}$ each) and stepwise (EPON/EtOH 1:1 and 2:1 each for $2 \mathrm{~h}$, EPON overnight) flat-embedded in EPON resin. Subsequently, EPON-embedded samples were polymerized for $24 \mathrm{~h}$ at $60^{\circ} \mathrm{C}$. After removal of the glass slide with $40 \%$ aqueous hydrofluoric acid, the EPON disk, containing the cells of interest, was removed from the dish, mounted on EPON resin blocks, and processed for ultramicrotomy. Serial sections were cut with a Leica EM UC7 ultramicrotome (Leica Microsystems) set to a thickness of $55 \mathrm{~nm}$. A final post staining of the ultrathin sections was perfomed for $6 \mathrm{~min}$ on a drop of $1 \%$ uranylacetate followed by $1 \%$ lead citrate for $3 \mathrm{~min}$ in aqueous solution.

Transmission electron microscopy (TEM) was conducted on a Jeol 1400FPlus (Tokyo, Japan) instrument at $120 \mathrm{kV}$ with magnifications as indicated. Digital images were acquired with a $4096 \times 4096$-pixel complementary metal-oxide semiconductor (CMOS) camera (TemCam-F416, TVIPS; Gauting, Germany). Postprocessing of the resulting 16-bit TIFF image files was performed with Fiji software version 1.52e (Image J). Pixel noise was averaged by a gaussian blur algorithm. A background resulting from inhomogeneous illumination was removed by implementing the "rolling ball" procedure, and the contrast was adjusted with histogram normalization.

Statistical analysis. If not mentioned otherwise, data are expressed as arithmetic mean \pm SEM and $n$ represents the number of mice. Student's $t$-test (two-tailed, if not indicated otherwise) in case of normal distribution or in case of multiple comparisons ANOVA, were used to detect statistically significant differences. $p$-Values of 0.05 or less were considered statistically significant. Statistical analyses and graphical presentations were computed with Graph Pad Prism software (Graph Pad, La Jolla, USA).
Reporting summary. Further information on research design is available in the Nature Research Reporting Summary linked to this article.

\section{Data availability}

The authors declare that data that support the findings of this study are available from the corresponding author upon reasonable request. Microarray data are deposited in the Gene Expression Omnibus (GEO) under the accession code GSE142175.

Received: 25 July 2019; Accepted: 17 February 2020;

Published online: 12 March 2020

\section{References}

1. Murray, P. J. \& Wynn, T. A. Protective and pathogenic functions of macrophage subsets. Nat. Rev. Immunol. 11, 723-737 (2011).

2. Honke, N. et al. Enforced viral replication activates adaptive immunity and is essential for the control of a cytopathic virus. Nat. Immunol. 13, 51-57 (2011).

3. Cervantes-Barragan, L. et al. Type I IFN-mediated protection of macrophages and dendritic cells secures control of murine coronavirus infection. J. Immunol. 182, 1099-1106 (2009).

4. Lang, P. A. et al. Tissue macrophages suppress viral replication and prevent severe immunopathology in an interferon-I-dependent manner in mice. Hepatology 52, 25-32 (2010).

5. Jenne, C. N. \& Kubes, P. Immune surveillance by the liver. Nat. Immunol. 14 996-1006 (2013).

6. Wardle, E. N. Kupffer cells and their function. Liver 7, 63-75 (1987).

7. Karasneh, G. A. \& Shukla, D. Herpes simplex virus infects most cell types in vitro: clues to its success. Virol. J. 8, 481 (2011)

8. Nicola, A. V. Herpesvirus entry into host cells mediated by endosomal low pH. Traffic 17, 965-975 (2016).

9. Agelidis, A. M. \& Shukla, D. Cell entry mechanisms of HSV: what we have learned in recent years. Future Virol. 10, 1145-1154 (2015).

10. Weed, D. J. \& Nicola, A. V. Herpes simplex virus membrane fusion. Adv. Anat. Embryol. Cell Biol. 223, 29-47 (2017).

11. Sodeik, B., Ebersold, M. W. \& Helenius, A. Microtubule-mediated transport of incoming herpes simplex virus 1 capsids to the nucleus. J. Cell Biol. 136 1007-1021 (1997).

12. Mabit, $H$. et al. Intact microtubules support adenovirus and herpes simplex virus infections. J. Virol. 76, 9962-9971 (2002).

13. Ojala, P. M., Sodeik, B., Ebersold, M. W., Kutay, U. \& Helenius, A. Herpes simplex virus type 1 entry into host cells: reconstitution of capsid binding and uncoating at the nuclear pore complex in vitro. Mol. Cell Biol. 20, 4922-4931 (2000).

14. Flatt, J. W. \& Greber, U. F. Viral mechanisms for docking and delivering at nuclear pore complexes. Semin. Cell Dev. Biol. 68, 59-71 (2017).

15. Yamauchi, Y. \& Greber, U. F. Principles of virus uncoating: cues and the snooker ball. Traffic 17, 569-592 (2016).

16. Virgin, H. W. The virome in mammalian physiology and disease. Cell 157, 142-150 (2014).

17. Bigalke, J. M. \& Heldwein, E. E. Nuclear exodus: herpesviruses lead the way Annu. Rev. Virol. 3, 387-409 (2016).

18. Mettenleiter, T. C. Herpesvirus assembly and egress. J. Virol. 76, 1537-1547 (2002).

19. Yamauchi, Y., Kiriyama, K., Kimura, H. \& Nishiyama, Y. Herpes simplex virus induces extensive modification and dynamic relocalisation of the nuclear mitotic apparatus (NuMA) protein in interphase cells. J. Cell Sci. 121, 2087-2096 (2008)

20. Lee, D. H. \& Ghiasi, H. Roles of M1 and M2 macrophages in herpes simplex virus 1 infectivity. J. Virol. 91, e00578-17 (2017).

21. Rasmussen, S. B. et al. Type I interferon production during herpes simplex virus infection is controlled by cell-type-specific viral recognition through Toll-like receptor 9, the mitochondrial antiviral signaling protein pathway, and novel recognition systems. J. Virol. 81, 13315-13324 (2007).

22. Leib, D. A. et al. Interferons regulate the phenotype of wild-type and mutant herpes simplex viruses in vivo. J. Exp. Med. 189, 663-672 (1999).

23. Piper, R. C. \& Katzmann, D. J. Biogenesis and function of multivesicular bodies. Annu. Rev. Cell Dev. Biol. 23, 519-547 (2007)

24. Trajkovic, K. et al. Ceramide triggers budding of exosome vesicles into multivesicular endosomes. Science 319, 1244-1247 (2008).

25. Ohanian, J. \& Ohanian, V. Sphingolipids in mammalian cell signalling. Cell Mol. Life Sci. 58, 2053-2068 (2001)

26. Spiegel, S. \& Merrill, A. H. Jr. Sphingolipid metabolism and cell growth regulation. FASEB J. 10, 1388-1397 (1996).

27. Tirodkar, T. S. \& Voelkel-Johnson, C. Sphingolipids in apoptosis. Exp. Oncol. 34, 231-242 (2012). 
28. Utermohlen, O., Herz, J., Schramm, M. \& Kronke, M. Fusogenicity of membranes: the impact of acid sphingomyelinase on innate immune responses. Immunobiology 213, 307-314 (2008).

29. Jimenez-Rojo, N., Garcia-Arribas, A. B., Sot, J., Alonso, A. \& Goni, F. M. Lipid bilayers containing sphingomyelins and ceramides of varying $\mathrm{N}$-acyl lengths: a glimpse into sphingolipid complexity. Biochim. Biophys. Acta 1838, 456-464 (2014).

30. Li, C. M. et al. The human acid ceramidase gene (ASAH): structure, chromosomal location, mutation analysis, and expression. Genomics $\mathbf{6 2}$ 223-231 (1999).

31. Zupancic, E., Carreira, A. C., de Almeida, R. F. \& Silva, L. C. Biophysical implications of sphingosine accumulation in membrane properties at neutral and acidic pH. J. Phys. Chem. B 118, 4858-4866 (2014).

32. Crameri, M. et al. $\mathrm{MxB}$ is an interferon-induced restriction factor of human herpesviruses. Nat. Commun. 9, 1980 (2018).

33. Nicola, A. V. \& Straus, S. E. Cellular and viral requirements for rapid endocytic entry of herpes simplex virus. J. Virol. 78, 7508-7517 (2004).

34. Dohner, K. et al. Importin alphal is required for nuclear import of herpes simplex virus proteins and capsid assembly in fibroblasts and neurons. PLoS Pathog. 14, e1006823 (2018).

35. Mikloska, Z. \& Cunningham, A. L. Alpha and gamma interferons inhibit herpes simplex virus type 1 infection and spread in epidermal cells after axonal transmission. J. Virol. 75, 11821-11826 (2001).

36. Kim, E. T., White, T. E., Brandariz-Nunez, A., Diaz-Griffero, F. \& Weitzman, M. D. SAMHD1 restricts herpes simplex virus 1 in macrophages by limiting DNA replication. J. Virol. 87, 12949-12956 (2013).

37. Gebai, A., Gorelik, A., Li, Z., Illes, K. \& Nagar, B. Structural basis for the activation of acid ceramidase. Nat. Commun. 9, 1621 (2018).

38. Goni, F. M. \& Alonso, A. Biophysics of sphingolipids I. Membrane properties of sphingosine, ceramides and other simple sphingolipids. Biochim. Biophys. Acta 1758, 1902-1921 (2006).

39. Nicola, A. V., McEvoy, A. M. \& Straus, S. E. Roles for endocytosis and low $\mathrm{pH}$ in herpes simplex virus entry into HeLa and Chinese hamster ovary cells. J. Virol. 77, 5324-5332 (2003).

40. Devadas, D. et al. Herpes simplex virus internalization into epithelial cells requires $\mathrm{Na}+/ \mathrm{H}+$ exchangers and p21-activated kinases but neither clathrin- nor caveolin-mediated endocytosis. J. Virol. 88, 13378-13395 (2014).

41. Cohen, G. H. et al. Structural analysis of the capsid polypeptides of herpes simplex virus types 1 and 2. J. Virol. 34, 521-531 (1980).

42. Fink, J. \& Seibel, J. Click reactions with functional sphingolipids. Biol. Chem. 399, 1157-1168 (2018)

43. Langlais, D., Barreiro, L. B. \& Gros, P. The macrophage IRF8/IRF1 regulome is required for protection against infections and is associated with chronic inflammation. J. Exp. Med. 213, 585-603 (2016).

44. Qi, C. F. et al. Differential expression of IRF8 in subsets of macrophages and dendritic cells and effects of IRF8 deficiency on splenic B cell and macrophage compartments. Immunol. Res. 45, 62-74 (2009).

45. $\mathrm{Hu}, \mathrm{X}$. et al. IRF8 regulates acid ceramidase expression to mediate apoptosis and suppresses myelogeneous leukemia. Cancer Res. 71, 2882-2891 (2011).

46. Molony, R. D. et al. Aging impairs both primary and secondary RIG-I signaling for interferon induction in human monocytes. Sci. Signal 10, eaan2392 (2017).

47. Stohlman, S. A., Woodward, J. G. \& Frelinger, J. A. Macrophage antiviral activity: extrinsic versus intrinsic activity. Infect. Immun. 36, 672-677 (1982).

48. Xu, Z., Tian, J., Smith, J. S. \& Byrnes, A. P. Clearance of adenovirus by Kupffer cells is mediated by scavenger receptors, natural antibodies, and complement. J. Virol. 82, 11705-11713 (2008).

49. Maler, M. D. et al. Key role of the scavenger receptor MARCO in mediating adenovirus infection and subsequent innate responses of macrophages. MBio 8, e00670-17 (2017).

50. Stichling, N. et al. Lung macrophage scavenger receptor SR-A6 (MARCO) is an adenovirus type-specific virus entry receptor. PLoS Pathog. 14, e1006914 (2018).

51. Le Blanc, I. et al. Endosome-to-cytosol transport of viral nucleocapsids. Nat. Cell Biol. 7, 653-664 (2005).

52. Nour, A. M., Li, Y., Wolenski, J. \& Modis, Y. Viral membrane fusion and nucleocapsid delivery into the cytoplasm are distinct events in some flaviviruses. PLoS Pathog. 9, e1003585 (2013).

53. Michen, B. \& Graule, T. Isoelectric points of viruses. J. Appl. Microbiol. 109, 388-397 (2010).

54. Olofsson, S. Isoelectric focusing of herpes simplex virus. Arch. Virol. 49, 93-98 (1975).

55. Jimenez-Rojo, N. et al. Membrane permeabilization induced by sphingosine: effect of negatively charged lipids. Biophys. J. 106, 2577-2584 (2014).

56. Hafez, I. M., Ansell, S. \& Cullis, P. R. Tunable pH-sensitive liposomes composed of mixtures of cationic and anionic lipids. Biophys. J. 79, 1438-1446 (2000).
57. Hollenbaugh, J. A. et al. Host factor SAMHD1 restricts DNA viruses in nondividing myeloid cells. PLoS Pathog. 9, e1003481 (2013).

58. Zhang, K., Lv, D. W. \& Li, R. Conserved herpesvirus protein kinases target SAMHD1 to facilitate virus replication. Cell Rep. 28, 449-459 e445 (2019).

59. Chow, A. Y. \& Mellman, I. Old lysosomes, new tricks: MHC II dynamics in DCs. Trends Immunol. 26, 72-78 (2005).

60. Geiger, T. et al. Initial quantitative proteomic map of 28 mouse tissues using the SILAC mouse. Mol. Cell Proteom. 12, 1709-1722 (2013).

61. Ehlert, K. et al. Farber disease: clinical presentation, pathogenesis and a new approach to treatment. Pediatr. Rheumatol. Online J. 5, 15 (2007).

62. Dropulic, L. K. \& Cohen, J. I. Severe viral infections and primary immunodeficiencies. Clin. Infect. Dis. 53, 897-909 (2011).

63. Rehwinkel, J. et al. SAMHD1-dependent retroviral control and escape in mice. EMBO J. 32, 2454-2462 (2013).

64. Sandbaumhuter, M. et al. Cytosolic herpes simplex virus capsids not only require binding inner tegument protein pUL36 but also pUL37 for active transport prior to secondary envelopment. Cell Microbiol. 15, 248-269 (2013).

65. Yamauchi, Y. et al. The UL14 tegument protein of herpes simplex virus type 1 is required for efficient nuclear transport of the alpha transinducing factor VP16 and viral capsids. J. Virol. 82, 1094-1106 (2008).

66. Krawczyk, A. et al. Overcoming drug-resistant herpes simplex virus (HSV) infection by a humanized antibody. Proc. Natl. Acad. Sci. USA 110, 6760-6765 (2013).

67. Henry, B. D. et al. Engineered liposomes sequester bacterial exotoxins and protect from severe invasive infections in mice. Nat. Biotechnol. 33, 81-88 (2015).

68. Walter, T. et al. Incorporation studies of clickable ceramides in Jurkat cell plasma membranes. Chem. Commun. (Camb.) 53, 6836-6839 (2017).

69. Subramanian, A. et al. Gene set enrichment analysis: a knowledge-based approach for interpreting genome-wide expression profiles. Proc. Natl. Acad. Sci. USA 102, 15545-15550 (2005).

70. Ashburner, M. et al. Gene ontology: tool for the unification of biology. The Gene Ontology Consortium. Nat. Genet. 25, 25-29 (2000).

71. Carpenter, A. E. et al. CellProfiler: image analysis software for identifying and quantifying cell phenotypes. Genome Biol. 7, R100 (2006).

72. Gulbins, A. et al. Antidepressants act by inducing autophagy controlled by sphingomyelin-ceramide. Mol. Psychiatry 23, 2324-2346 (2018).

73. Kachler, K. et al. Enhanced acid sphingomyelinase activity drives immune evasion and tumor growth in non-small cell lung carcinoma. Cancer Res. 77, 5963-5976 (2017)

\section{Acknowledgements}

We thank Alexandra Brenzel and Sylvia Voortmann from the Imaging Center Essen (IMCES) for their technical support. We further thank Daniel Herrmann for technical assistance with the mass spectrometric analyses and Michael Möllmann for help with cell sorting. Images shown in this publication were acquired on IMCES instruments. This study was funded by the German Research Council (DFG - Deutsche Forschungsgemeinschaft; GRK2098, GRK2581, SFB974, GRK1949, GU 335/35-1, LA1419/71, LA1419/10-1, HU1824/7-1, SE1410/6-2 and SFB1292/TP13), the Fresenius Stiftung (2015_A232, MH), GIF (I-1474-414,13/2918, MH) and the EU 7th framework, MarieCurie Actions ITN-EDGE (https://ec.europa.eu/research/mariecurieactions/about/ innovative-training-networks to BS). Justa Friebus-Kardash was supported by the "Programm zur internen Forschungsförderung Essen" (IFORES) Research fellowship program of the University of Duisburg-Essen Medical School. We acknowledge support by the Open Access Publication Fund of the University of Duisburg-Essen.

\section{Author contributions}

Conceptualization, J.L., E.G., K.S.L.; Investigation J.L., A.R.N., V.D., S.K.F., H.B., F.C., P.B., J.F., M.B., D.O., K.A.B., J.F.K., E.G., K.S.L.; Formal analysis J.L., E.G., K.S.L.; Investigation and formal analysis electron microscopy, J.L., H.J., B.W.; Investigation and formal analysis mass spectrometry F.S., B.K.; Investigation and formal analysis click chemistry J.L., J.F., J.S.; Investigation and formal analysis microarray J.L., P.B., K.K.; Writing - original draf J.L., K.S.L.; Writing - review \& editing J.L., M.J.E., P.A.L., E.G., B.S., H.J., J.S., U.F.G., K.S.L., C.H., A.K., E.B.B., J.R.G., B.K., F.S.; Resources K.S.L., P.A.L., E.G., E.B.B., U.F.G. B.S., J.F., A.K., B.K., M.H., A.B., H.C.P.; Supervision K.S.L., E.G., U.F.G., B.S.

\section{Competing interests}

J.L., M.J.E., E.G., and K.S.L. declare that they have applied for a patent related to the treatment of viral infections with sphingosine. Patent applicant: Harbins Ruhr Bioscience; name of inventors: Erich Gulbins, Karl Lang, Judith Lang; application number: DE 102018217334.6 (German), 57HARB10052WO 16/588,361 (USA); status of application: pending; specific aspect of manuscript covered in patent application: claims on the basis of data shown in Fig. 3c, h, i. The remaining authors declare no competing interests. 


\section{Additional information}

Supplementary information is available for this paper at https://doi.org/10.1038/s41467020-15072-8

Correspondence and requests for materials should be addressed to K.S.L.

Peer review information Nature Communications thanks JIllian Carr and the other, anonymous, reviewer(s) for their contribution to the peer review of this work.

Reprints and permission information is available at http://www.nature.com/reprints

Publisher's note Springer Nature remains neutral with regard to jurisdictional claims in published maps and institutional affiliations. (c) Open Access This article is licensed under a Creative Commons Attribution 4.0 International License, which permits use, sharing, adaptation, distribution and reproduction in any medium or format, as long as you give appropriate credit to the original author(s) and the source, provide a link to the Creative Commons license, and indicate if changes were made. The images or other third party material in this article are included in the article's Creative Commons license, unless indicated otherwise in a credit line to the material. If material is not included in the article's Creative Commons license and your intended use is not permitted by statutory regulation or exceeds the permitted use, you will need to obtain permission directly from the copyright holder. To view a copy of this license, visit http://creativecommons.org/licenses/by/4.0/.

(C) The Author(s) 2020 